\title{
RAM-589.555 favors neuroprotective and anti-inflammatory profile of CNS-resident glial cells in acute relapse EAE affected mice
}

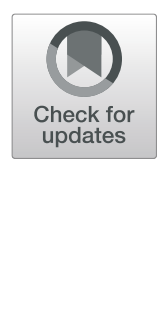

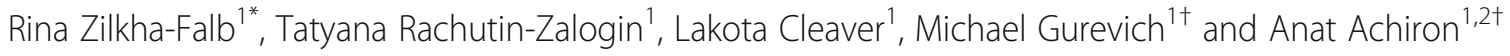

\begin{abstract}
Background: Targeting RNA polymerase-1 (POL1) machinery is a new strategy for suppression of multiple sclerosis (MS) relapse activity. Oral administration of POL1 inhibitor RAM-589.555, which is characterized by high permeability and bioavailability in naïve mice, ameliorates proteolipid protein (PLP)-induced experimental autoimmune encephalomyelitis (EAE) by suppressing activated autoreactive lymphocytes. We assessed the accessibility of RAM589.555 to the central nervous system (CNS) of EAE-mice and further investigated its immunomodulatory effects on CNS-resident astro- and micro-glial cells in-vitro and in-vivo.

Methods: Effects of RAM-589.555 on activated microglia and astrocyte viability, proliferation, and secretion of neurotrophic factors were assessed in-vitro. The pharmacokinetic of RAM-589.555 was evaluated in the blood and central nervous system (CNS) of EAE-affected mice. High-dimensional single-cell mass cytometry was applied to characterize the effect of RAM-589.555 on EAE-affected mice's CNS-resident micro- and astroglial cells and CNS-infiltrating immune cells, which were obtained seven days after RAM-589.555 administration at EAE onset. Simultaneously, the expression level of pre-rRNA, the POL1 end product, was assessed in blood cells, microglia, and astrocytes to monitor RAM-589.555 effects.
\end{abstract}

Results: RAM-589.555 demonstrated blood and CNS permeability in EAE mice. In-vitro, incubation with 400 nM of RAM589.555 significantly reduced viability and proliferation of lipopolysaccharide (LPS)-activated microglia by $70 \%$ and $45 \%$ ( $p$ $<0.05)$, respectively, while tumor necrosis factor a (TNFa)-activated astrocytes were not affected. The secretion of neurotrophic factors was preserved. Furthermore, 7 days after administration of RAM-589.555 at EAE onset, the level of pre-rRNA transcript in peripheral blood mononuclear cells (PBMC) was decreased by $38.6 \%(p=0.02)$, while levels of prerRNA transcript in microglia and astrocytes remained unchanged. The high-dimensional single-cell mass cytometry analysis showed decreased percentages of CNS-resident microglia and astrocytes, diminished pro-inflammatory cytokines (IL-1 $\beta, I L-6, I L-12, I L-17, T N F a$, and IFN $\gamma$ ), and an increase of their anti-inflammatory cytokines (IL-4, IL-10, and TGF $\beta$ ) in RAM-589.555-treated compared to vehicle-treated mice $(p<0.05)$.

Conclusions: These data correlate RAM-589.555-induced clinical amelioration and its CNS-permeability to decreased CNSinflammation, and decreased micro- and astrogliosis, while restoring micro- and astroglial anti-inflammatory and neuroprotective capacity.

Keywords: Multiple sclerosis, Polymerase-1 inhibitor, Experimental autoimmune encephalomyelitis, Microglia, Astrocytes, High-dimensional single-cell mass cytometry

\footnotetext{
* Correspondence: rina.falb@sheba.health.gov.il

${ }^{\dagger}$ Michael Gurevich and Anat Achiron contributed equally.

${ }^{1}$ Neuroimmunology Laboratory, Multiple Sclerosis Center, Sheba Medical

Center, Ramat Gan, Israel

Full list of author information is available at the end of the article
}

\section{$\triangle B M C$}

(c) The Author(s). 2020 Open Access This article is licensed under a Creative Commons Attribution 4.0 International License, which permits use, sharing, adaptation, distribution and reproduction in any medium or format, as long as you give appropriate credit to the original author(s) and the source, provide a link to the Creative Commons licence, and indicate if changes were made. The images or other third party material in this article are included in the article's Creative Commons licence, unless indicated otherwise in a credit line to the material. If material is not included in the article's Creative Commons licence and your intended use is not permitted by statutory regulation or exceeds the permitted use, you will need to obtain permission directly from the copyright holder. To view a copy of this licence, visit http://creativecommons.org/licenses/by/4.0/ The Creative Commons Public Domain Dedication waiver (http://creativecommons.org/publicdomain/zero/1.0/) applies to the data made available in this article, unless otherwise stated in a credit line to the data. 


\section{Introduction}

Multiple sclerosis (MS), the most common demyelinating disease of the central nervous system (CNS) affecting young adults, is manifested by various combinations of neurological symptoms including motor, sensory, coordination, visual, and cognitive modalities, that lead to significant disability over time [1-3]. MS is considered a T cell-mediated autoimmune disease ensuing from attacks of peripheral adaptive immune system ( $\mathrm{T}$ and $\mathrm{B}$ lymphocytes) and provoked by the peripheral innate immune system (macrophages, dendritic cells, natural killer cells, etc.).

Recent studies have suggested that the CNS-residing innate immune cells (microglia and astrocytes) also play an important role both in initiation and progression of MS by influencing the effector function of $\mathrm{T}$ and $\mathrm{B}$ cells $[4,5]$. The effector cells, in turn, express cytokines and activation markers that further activate innate immune cells [6]. Microglia are activated in MS and can secrete pro-inflammatory cytokines, reactive oxygen intermediates, proteinase, and complement proteins. Microglia also have neuroprotective phenotypes. However, microglia lose their normal homeostatic phenotype in MS. Astrocytes are involved in multiple aspects of CNS function, including synapse maintenance, neurotransmission, phagocytosis, and blood-brain barrier formation. Nevertheless, neurotoxic subpopulation of astrocytes (termed A1 astrocytes) has been described [7], and the primary driver of A1 astrocytes are microglia. Astrocytes promote MS by producing neurotoxic molecules, such as nitric oxide (NO) and TNF $\alpha$, and by recruiting neurotoxic inflammatory monocytes to the CNS.

Particularly, astrocytes and microglia are major players of myelin production both in normal and pathological conditions [8], endowed with a pro-regenerative trophic factors support, and recruit oligodendrocyte progenitor cells (OPCs) to the demyelinating area to further differentiate into myelinating oligodendrocytes (reviewed in details elsewhere [9-11]).

An array of neurotrophic factors (NFs) secreted by astrocytes and microglia is important for the maintenance of neurons and oligodendrocytes during normal homeostasis, and in CNS pathogenesis, demyelinating models, particularly in EAE and even in the MS tissue, these include brain-derived neurotrophic factor (BDNF), CNTF, NGF, glial-derived neurotrophic factor (GDNF), and fibroblast growth factor basic (FGF2) [9, 12-14]. Obviously, other astro- and microglial-secreted proteins negatively regulate oligodendrocyte biology and play detrimental role. Hence, under pathological and demyelinating conditions, reactive astrocytes and activated microglia both exhibit a dual activity-inducing detrimental or beneficial effects, the balance of which appears to be critical for tissue regeneration [8].
An effective treatment of progressive forms of MS will depend on the early treatment of inflammation to prevent compartmentalized inflammation developing in the CNS, treatment that targets innate immune processes in the brain, and non-immune approaches that target neurodegeneration, axonal dysfunction, and promote myelin repair [15].

Previously, we have demonstrated that the geneexpression profile in peripheral blood mononuclear cells (PBMC) from MS patients with active disease is associated with significant over-activation of the RNA polymerase 1 (POL1) molecular pathway. We further demonstrated that decreased expression of key POL1related genes (RRN3, POLR1D, LRPPRC) is associated with suppression of inflammation by downregulation of NFkB and activation of the p53-dependent apoptotic mechanism [16]. The association of POL1 machinery with MS pathogenesis was confirmed by the therapeutic efficacy of silencing three POL1-related genes in MOG-primed lymph node cells (LNCs) in a model of passively induced experimental autoimmune encephalomyelitis (EAE) [17]. From human perspective, this therapeutic strategy that addresses the rapid proliferation of autoreactive inflammatory cells raised from searching beneficial mechanism attenuating MS progression.

Moreover, we previously demonstrated that administration of RAM-589.555, a specific POL1 inhibitor (demonstrated in cell-free based assay) suppressed EAE induction and reduced disease severity [18, 19]; hence, we proposed the POL1 pathway as a potential target for therapeutic interventions not only in RRMS but also during progressive episodes. Our further study of RAM589.555 demonstrated that it had medium-high permeability and bioavailability after oral gavage in naïve mice [19]. These observations prompted us to investigate whether RAM-589.555 can cross the blood-brain barrier (BBB) and access the CNS in EAE-displaying mice and further investigate its immunomodulatory effects on CNS-resident glial cells.

In the current study, we have assessed the accessibility of RAM-589.555 to the CNS of EAE-mice and further investigated its immunomodulatory effects on CNS-resident glial cells in-vitro and in-vivo. Our results show that RAM-589.555 accesses the CNS, reduces the number of infiltrating immune cells, and reduces the number of microglia and astrocytes while bolstering their anti-inflammatory cytokine profile. These findings suggest that the therapeutic mechanism underlying the amelioration of relapsing-remitting EAE by RAM-589.555 is associated with decreased CNS inflammation and, most importantly, restoration of the neuroprotective and anti-inflammatory capacity of CNS-resident microglia and astrocytes. 


\section{Methods}

This study included in-vivo and in-vitro experimental arms. Briefly, the in-vivo arm (Fig. 1a) included a disease model in mice with RAM-589.555 treatment, pharmacokinetic analysis of RAM-589.555, single-cell mass cytometry, evaluation of pre-rRNA transcript level, and histopathological examination. The in-vitro arm (Fig. 1b) included the preparation of primary micro- and astroglial cultures, subsequent exposure to RAM-589.555, and assessment of cellular phenotype, viability, proliferation, pre-rRNA transcript, and secretion of NFs.

\section{Animals}

The postnatal day 3 (P3) CD-1 and adult 8-week-old female SJL/J mice used in this study were obtained from Envigo (Jerusalem, Israel); they were maintained in the pathogen-free animal facilities of Sheba Medical Center (Tel- Hashomer, Israel). For the in-vitro and in-vivo experiments, 18 neonate pups of CD-1 strain and 68 mice of SJL/J strain were used, respectively. All animal procedures and experimental protocols were approved by the Israel Animal Care and Use Committee (IACUC) of Sheba Medical Center (permit number 1054/16) and were performed in compliance with its relevant guidelines and regulations. The P3 CD-1 mice were used only for isolation of primary astrocytes and microglia for the in-vitro experiments following a previous published protocol [20]. The need to use CD-1 strain neonates for in-vitro experiments raised from technical difficult as lactating mice are aggressive and use to harm their offspring after delivery while these of the CD-1 strain is more gentle; consequently, the neonates survive.

The SJL/J mice served for in-vivo experiments are well known susceptible mice for EAE induction with PLP139-151. Obviously, the results were not impacted by the different mice strains as they were used independently for separate sets of experiments.

\section{Culture of microglia and astrocytes, stimulation, and imaging}

Microglial and astroglial cells were isolated from brain cortices of P3 mice. Briefly, brains were dissected, weighed, and enzymatically digested using Neural Tissue Dissociation Kit (\# 130-092-628 for microglia and \# 130093-231 for astrocytes, Miltenyi Biotec, Germany) for 35 min at $37{ }^{\circ} \mathrm{C}$ following previous protocol [20]. Further processing was performed at $4{ }^{\circ} \mathrm{C}$. Tissue debris was removed by passing the cell suspension through a $70 \mu \mathrm{m}$ cell strainer. Microglial and astroglial cells were magnetically labeled with $\mathrm{CD} 1 \mathrm{~b}^{+}$[as described [21]; anti CD11b MicroBeads kit, Miltenyi Biotec, \#130-093-636] and GFAP $^{+}$microbeads [as described [20]; anti-GLAST (ACSA-1) MicroBeads kit, Miltenyi Biotec, \#130-095825], respectively and loaded onto a MACS $^{\circledR}$ column
(Miltenyi Biotec, Germany), which was placed in the magnetic field of a MACS Separator (Miltenyi Biotec, Germany). The amounts of antibodies and magnetic beads were calculated based on the number of cells obtained after myelin removal (Myelin removal beads II \# 130-096-733, Miltenyi Biotec, Germany), using the manufacturer's guidelines. Single-cell suspension was plated $\left(125,000\right.$ cells $\left./ \mathrm{cm}^{2}\right)$ in 6-wells tissue-culture plate (Greiner Bio-One Cellstar ${ }^{\oplus}$ Sigma-Aldrich, Rehovot, Israel) precoated with poly-D-lysine [PDL (Sigma-Aldrich, Rehovot, Israel)], in growth medium [Dulbecco's modified Eagle's medium (DMEM) (Invitrogen, Carlsbad, USA)], supplemented with 10\% serum (Invitrogen, Carlsbad, USA), penicillin/streptomycin, and glutamine. Fresh media was added every 2 days to maintain proliferation of cells, which were then passaged every 5 days and re-plated as single cells using trypsin (Sigma-Aldrich, Rehovot, Israel) for continued growth until $3 \times 10^{6}$ cells were available for in-vitro assays ( $\sim 5$ passages, i.e., approximately 25 days). The cell viability was examined immediately after magnetic separation of the freshly isolated cells and during passages. Live and dead cells were distinguished by $\mathrm{NC}$ 100 Nucleocounter (ChemoMetec A/S, Allerod, Denmark). Approximately, 97\% of $\mathrm{GLAST}^{+}$astrocytes or $\mathrm{CD}_{11 b^{+}}$microglia are viable.

For detection of viability, proliferation, and secretion of neurotrophic factors, microglia and astrocytes were plated in serum-free medium (SFM; DMEM supplemented with B27) and activated with LPS (100 ng/ml) for microglia or TNF $\alpha(100 \mathrm{ng} / \mathrm{ml})$ for astrocytes and in the absence or presence of elevated concentrations of RAM-589.555 (25-400 nM) on poly-D-lysine [PDL (Sigma-Aldrich, Rehovot, Israel)] coated 96-well plate (200,000 cells/well). After $48 \mathrm{~h}$, cells and supernatants were collected. For immunocytochemistry, cells were plated on PDL-coated coverslips and analyzed by fluorescent microscopy.

\section{Brain-derived neurotrophic factor, fibroblast growth factor basic, and glial-derived neurotrophic factor ELISA} Levels of brain-derived neurotrophic factor (BDNF), glial-derived neurotrophic factor (GDNF), and fibroblast growth factor basic (FGF2) were measured by the enzyme-linked immunosorbent assay (ELISA; Quantikine ELISA total BDNF; DBNT00 and FGF basic; MFB00; R\&D systems, Abingdon, UK and mouse GDNF ELISA Kits; EA100590; OriGene, Rockville, MD, USA, respectively) according to the manufacturer's instructions. Samples were tested in duplicate and the mean was calculated. The intra- and interassay coefficients of variation for BDNF were $<3 \%$ and $<7 \%$, for GDNF $<6 \%$ and $<8 \%$, and for FGF2 $<3 \%$ and $<8 \%$, respectively. Results are the average of three independent repeats. 
a

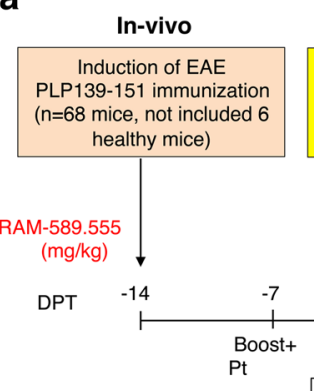

clinical follow-up

\section{-up}

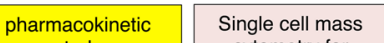

study cytometry for

point)
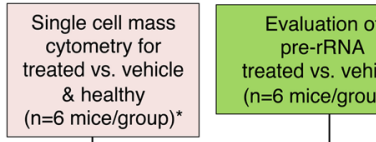

pre-rRNA

( $\mathrm{n}=6$ mice/group) ${ }^{*}$

histopathological

vs. vehicle ( $n=3$

mice/group)
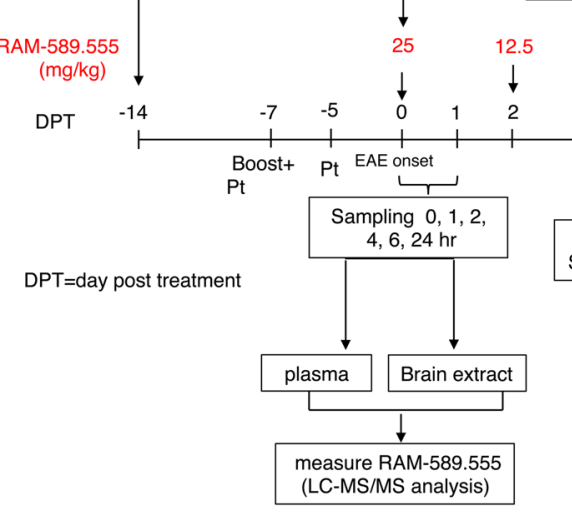

mice/group $)^{*}$

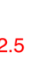

\section{5}
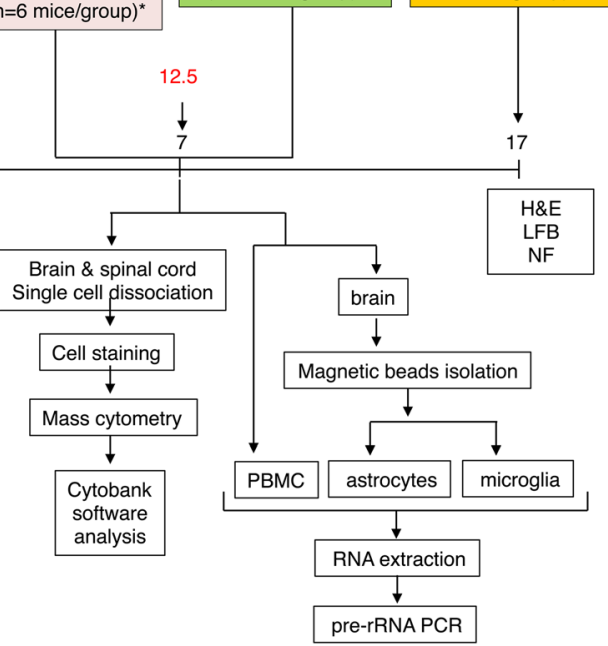

b

$$
\text { In-vitro }
$$

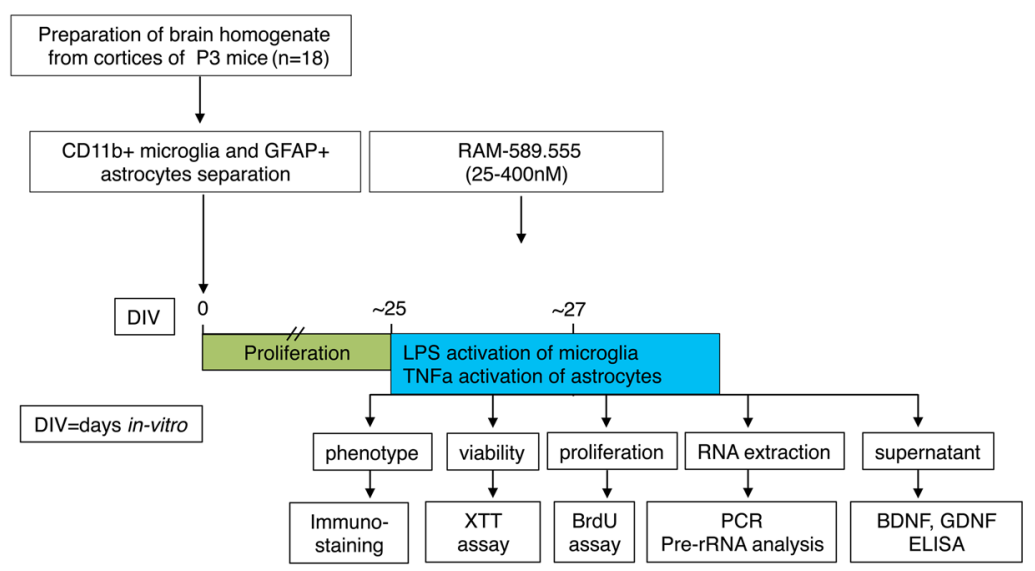

Fig. 1 Scheme of experimental design of the study. a Set of in-vivo experiments in CD1 1 b microglia and GFAP ${ }^{+}$astrocytes. $\mathbf{b}$ Set of in-vitro experiments

\section{Monitoring cell viability and proliferation}

Cell viability was monitored using an XTT cell viability assay kit (Biological Industries Israel Beit-Haemek Ltd., Kibbutz Beit-Haemek, Israel) following the protocols described by the manufacturer. After culturing for $2-4 \mathrm{~h}$ at $37^{\circ} \mathrm{C}$ and then agitating plates gently for $5 \mathrm{~min}$, the absorbance was read at $450 \mathrm{~nm}$ using a 96-well plate reader (Tecan Sunrise absorbance reader; Tecan UK) and proliferation was monitored by BrdU assay as previously described [19].

\section{Mice, EAE model, and RAM-589.555 administration}

SJL/J mice were injected subcutaneously at one site in the flank with PLP 139-151 [150 $\mu \mathrm{g} /$ mouse $+300 \mu \mathrm{g}$ Mycobacterium tuberculosis H37Ra (Mt; Difco, Detroit,
MI)] and another boost a week later in the other flank [22]. At the day of boost and $48 \mathrm{~h}$ later (Fig. 1a), mice received $300 \mathrm{ng}$ of pertussis toxin in $500 \mu \mathrm{l}$ of PBS per mouse (by intraperitoneal injection). RAM-589.555 treatment by oral gavage was initiated at the onset of the disease $(25 \mathrm{mg} / \mathrm{kg})$, approximately at day 14 after the first PLP139-151 injection, and then re-administered at $48 \mathrm{~h}$ and 1 week after disease onset (both with $12.5 \mathrm{mg} /$ kg) (Fig. 1a). Vehicle (water)-treated, age-matched mice (8 weeks old) served as controls. Each group included 10 mice. Mice were allocated pairwise to control and RAM589.555 treatment groups according to their clinical scores, allowing an identical distribution of EAE scores in both groups at the onset of treatment. Mice were scored daily for clinical signs of EAE as described 
previously [19]: 1-flaccid tail, 2-hind limbs paraparesis, 3-hind limbs paralysis, 4-quadriplegia. In order to optimize the experiment setting, RAM-589.555 was administered exclusively when each mouse received score 1. The level of pre-rRNA transcript in-vivo was assessed a week after initial RAM-589.555 gavage, and before administrating its third dose. Mice were bled from tail vein ( $n=6$ mice/group) into heparinized tubes and brains were subjected for microglia and astrocyte magnetic beads isolation. Blood was subjected to red blood cell (RBC) lysis and cell pellets were resuspended in $350 \mu \mathrm{l}$ Purelink kit lysis buffer (Invitrogen). Similarly, isolated microglia and astrocytes were re-suspended in the lysis buffer for RNA extraction, cDNA preparation, and quantitative real-time PCR as described previously [19]. The remaining mice were followed up until 17 days post treatment (DPT) and subjected to histopathology analysis (Fig. 1a).

\section{Dosing regimen}

The choice of RAM-589.555 dosing scheme and doses are based on our previous studies in MOG-35-55 induced EAE $[18,19]$ where different doses and administration schemes were tested. Dosing studies showed that administration of high dose POL1-inhibitor (POL1-I; 50 $\mathrm{mg} /$ mouse, daily, for 10 consecutive days) to C57BL/6J mice resulted in high mortality rates. It is for this reason that we lowered the dose to $12.5 \mathrm{mg} /$ mouse daily for 10 consecutive days, which preserved survival. Using this dose, we were able to demonstrate that this treatment regimen was effective in delaying the onset and reducing the overall severity of EAE as demonstrated in significantly low EAE clinical and cumulative scores, when treatment was administered before disease induction (immunization model) as well as when administered after the appearance of clinical signs (treatment model), as compared to vehicle-treated animals [18].

After single $12.5 \mathrm{mg} / \mathrm{kg}$ RAM-589.555 administration, its level in blood remains considerably high [19]; this mean that in order to preserve its blood level without exceeding its tolerated concentration, RAM-589.555 can be administered in intervals. Following our experience with POL1-I-suppressing EAE and POL1-I -treatment $(12.5 \mathrm{mg} / \mathrm{kg})$ in the chronic EAE model, we determined the initial $25 \mathrm{mg} / \mathrm{kg}$ (at the onset of EAE) followed by $12.5 \mathrm{mg} / \mathrm{kg}$ after $48 \mathrm{~h}$ and 7 days later.

With regard to toxicology, levels of $>50 \mathrm{mg} / \mathrm{kg}$ are toxic and we further characterized the therapeutic range without toxic effect.

Previously, in a preliminary set of experiments in healthy mice, we assessed the toxicity of oral administration of a single dose of RAM-589.555 that was tested in naïve mice using range from 3 to $500.0 \mathrm{mg} / \mathrm{kg}$. No effect on survival or behavioral traits was observed at $24 \mathrm{~h}$.
Indeed, RAM-589.555 was well tolerated when administered to EAE mice every alternate day until disease onset at a dose of $30 \mathrm{mg} / \mathrm{kg}$. In these previous studies, we also examined EAE mice survival in the EAE immunization model for the following oral daily POL1-I doses, for 10 consecutive days: $50.0 \mathrm{mg} / \mathrm{kg}, 25.0 \mathrm{mg} / \mathrm{kg}$, and $12.5 \mathrm{mg} /$ $\mathrm{kg}(n=6$ / group) or vehicle $(n=7)$. The survival rate in the vehicle group was $85.7 \%$; in comparison, the survival rate using daily dose of $50.0 \mathrm{mg} / \mathrm{kg}$ POL1-I was $0 \%$, daily dose of $25.0 \mathrm{mg} / \mathrm{kg}$ resulted in $83.3 \%$ survival, and using a daily dose of $12.5 \mathrm{mg} / \mathrm{kg}$ resulted in $100 \%$ survival, during 5 weeks of follow-up. Accordingly, daily dose of $12.5 \mathrm{mg} / \mathrm{kg}$ was chosen for further experiments. We estimated that single first administration of $25 \mathrm{mg} / \mathrm{kg}$ is somewhat equivalent to two intermittent administration of $12.5 \mathrm{mg} / \mathrm{kg}$ that can be followed with two $12.5 \mathrm{mg} / \mathrm{kg}$ doses after $48 \mathrm{~h}$ and 7 days later.

\section{qRT-PCR analysis}

cDNA was subjected for qRT-PCR in triplicates using the 7500 Real-Time PCR system (Applied Biosystems, Foster City, CA, USA) and TaqMan Universal PCR Master Mix (Applied Biosystems). Relative expression was calculated by Relative Quantification software (Roche Diagnostics). $\beta$-Actin was used as an internal control to normalize target gene expression levels, which were determined with the $2^{-\Delta C T}$ method. Results are the average of three independent repeats.

Primers sequences used for amplification were as follows:

For the pre-rRNA: forward: tttcttgtaagcgtcgaggtg, reverse: agcaggcacctaggagacaa; (Sigma, Israel) with Probe ID: \#1, cat. no. 04684974001 (Roche, Switzerland).

$\beta$-Actin: TaqMan Real-Time ready assay ID: Mm00607939_s1.

For in vitro experiment, the results present the expression of pre-rRNA transcript relative to $\beta$-actin. For invivo experiments, the results present the fold change (FC) compare pre-rRNA transcript in healthy mice.

\section{Histopathology, immunohistochemistry, inflammation, and demyelination}

Histological evaluation was done on paraformaldehydefixed, paraffin-embedded sections of spinal cords (SCs). Sections were stained with hematoxylin and eosin (HE), Luxol fast blue (LFB), and neurofilament (NF) [3] staining to assess inflammation, demyelination, and axonal pathology, respectively, as described previously [18]. We examined 12-16 longitudinal sections per mouse. Quantitative histological evaluation for inflammation and demyelination and axonal loss was done and scored blindly by two independent observers. Histopathology analysis was done at day 17 after start of RAM-589.555 treatment. Mice $(n=3)$ from each group received ketamine/ 
xylazine and were perfused transcardially with PBS followed by $4 \%$ paraformaldehyde (PFA) in PBS. Spinal cords were removed and stored in $4 \%$ PFA at $4{ }^{\circ} \mathrm{C}$. Longitudinal spinal cord sections were performed, stained by hematoxylin \& eosin (H\&E), Luxol fast blue (LFB), and neurofilament staining (NF, mouse anti-human NF protein, Dako) in $6 \mu \mathrm{m}$ paraffin-embedded adjacent serial sections. The morphometric quantification of inflammation, demyelination, and axonal damage were performed using Olympus IX-73 microscope coupled to imaging software (Cellsens Entery digital imaging software, Olympus). The following parameters were evaluated: (a) inflammation: average number of lymphocyte infiltrations per square millimeter counted in spinal cord sections using a grid overlay (H\&E); (b) demyelination: mean percent of demyelination areas per square millimeter in spinal cord sections (LFB); and (c) axonal damage: mean percent of axonal loss assessed by neurofilament loss per square millimeter in spinal cord sections. Both the cervical, thoracic, and lumbar regions were assessed. Quantitative analysis of immunopositive or stained region was carried out using NIH Image (ImageJ 1.43u).

\section{Pharmacokinetic measurements}

Mice that were induced for EAE and randomly assigned to a RAM-589.555 treatment, as described above, were sampled (4 mice per time point) at $0,1,2,4,6$, and $24 \mathrm{~h}$ after the initial $25 \mathrm{mg} / \mathrm{kg}$ RAM-589.555 treatment at EAE onset. Mice were bled from tail vein into heparin drop containing tube then and perfused with ice-cold PBS to ensure that only parenchyma-derived RAM- 589.555 was assessed and the brain was isolated. Plasma and brain samples were maintained at $-80{ }^{\circ} \mathrm{C}$ until RAM-589.555 was tested by liquid chromatography-tandem mass spectrometry (LC-MS/MS) analysis. The Waters Acquity HClass FTN UPLC and MS/MS detector: Waters Xevo TQ$\mathrm{S}$ micro was used. The area under the curve to the last measurable concentration $\left(\mathrm{AUC}_{0-\mathrm{t}}\right)$ was calculated by the trapezoidal rule method [23].

\section{Immunocytochemistry}

Coverslips were washed with PBS, fixed with $2 \%$ PFA, and immune-labeled as described previously [24], except for the primary antibodies used, which were rabbit antiCD11b (1:200; Abcam) and monoclonal mouse antiGFAP (1:500; Sigma-Aldrich). Slides were examined for expression of markers using an IX-73 fluorescence microscope (Olympus, Tokyo, Japan) with magnifications $\times 10$ or $\times 20$. Digital images were acquired and analyzed using a digital camera system (DP74, Olympus, Tokyo, Japan) coupled to imaging software (Cellsens Entery digital imaging software, Olympus) or confocal LSM710.

\section{Brain and spinal cord single-cell dissociation}

Mice were anesthetized and perfused with ice-cold PBS. Brains and SCs were dissected and after removal of meninges, and digested as described previously [25]. Cell suspensions were subjected to myelin removal using myelin removal beads II kit (cat. 130-096-733; Miltenyi Biotec) and cell pellets were suspended in $1 \mathrm{ml}$ of staining buffer (PBS containing $2 \%$ bovine serum albumin and $0.09 \%$ sodium azide) and then subjected for mass cytometry (see experimental procedure in Additional file 1). The perfusion quality was evaluated as previously described [2628 ]. Briefly, cells in the blood circulation were labeled by injecting the PE-conjugated anti-CD45 antibody (BioLegend) to the tail vein of mice $(3 \mu \mathrm{g} /$ mouse, in $150 \mu \mathrm{l}$ of PBS). Thus, $97 \%$ of the immune cells in the circulation were labeled and following perfusion enabling us to identify the cells that originated from the blood and present in CNS compartment by their PE-conjugated anti-CD45 antibody labeling. To distinguish the CNS-resident immune cells from these originated from the blood, we stained the cells extracted from the CNS with an APCconjugated anti-CD45 antibody (Biolegend). CNS compartment contained $4.2 \%$ immune cells, while only $0.5 \%$ blood-derived immune cells (Additional file 2).

\section{Mass cytometry}

Purified CNS-single cells $\left(3 \times 10^{6}\right.$ per sample) were first incubated with FcR blocking reagent (cat. 130-092-575; Miltenyi Biotec) according to manufacturer's protocol and then stained $\left(100-\mu \mathrm{l}\right.$ final staining reaction volume; $\left.1 \mathrm{~h} ; 4^{\circ} \mathrm{C}\right)$ with a mixture of metal-tagged anti-cytokine/chemokine/growth antibodies (a complete list of antibodies is provided in Additional table 1) conjugated using the MAXPAR reagent (Fluidigm Inc.). Rhodium (1:2,000; Fluidigm Inc.) was added to the cells in the last $20 \mathrm{~min}$ of staining. Cells were then washed twice with staining buffer, fixed in 1.6\% PFA (Sigma-Aldrich) in PBS (1 h, RT), stained with iridium (1:2, 000, $20 \mathrm{~min}, \mathrm{RT}$; Fluidigm Inc.), washed again in ultrapure $\mathrm{H}_{2} \mathrm{O}$ (to prevent cell loss, the sample was centrifuged at 10 , $000 \mathrm{~g}$ for $1 \mathrm{~min}$ ), and analyzed on a CyTOF I machine (Fluidigm Inc.), with events acquired at approximately 500 events per second. Internal metal-isotope bead standards were added for sample normalization. Acquired data were uploaded to a Cytobank webserver (Cytobank Inc.) for data processing and for gating out of dead cells and normalization of beads. Rhodium (Rh) and iridium (Ir) (Fluidigm Inc.) inter-chelators were used to identify live/dead cells. At least 20,000 live single cells were analyzed in each sample. The $\mathrm{Rh}$ gating was used to assess live/dead cells [29].

\section{Mass cytometry data analysis}

Data were transformed on Cytobank using an arcsinh (X/5) transformation. Spanning-tree progression analysis of density-normalized events (SPADE) analyses in 
Cytobank was performed with the target number of nodes set to 100 and sampling $100 \%$ of events. In SPADE, the color gradient indicates the median expression level of the chosen marker. For t-distributed stochastic neighbor embedding ( $t$-SNE)-based visualization (viSNE) analyses, all cells were chosen for sampling. Data analysis was performed using SPADE21 and viSNE20 algorithms on gated live single cells (see gating strategy for mass cytometry single-cell analysis in Additional file 3). In both algorithms, the following markers were chosen for clustering: CD45, CX3CR1, CD11b, and GFAP. Cell populations were then defined based on marker expression distribution in the spade figure, according to standard definitions of cell types: microglia, $\mathrm{CD} 45^{\text {low }} \mathrm{CD} 11 \mathrm{~b}^{+}$; astrocytes, $\mathrm{CD} 45^{\text {neg }} \mathrm{GFAP}^{+}$; and infiltrating immune cells, $\mathrm{CD} 45^{\text {high }}$. Intensity levels of markers were calculated on transformed $(\operatorname{arcsinh}(\mathrm{X} / 5))$ median intensity values in each defined cell population.

\section{Statistical analysis}

Data was presented as mean \pm SEM. The number of experimental repeats used for each analysis was presented in the appropriate figure legend. All statistical analyses were conducted using SPSS version 22 (IBM). EAE clinical scores were analyzed using Mann-Whitney test. Quantitation of pathology, mass cytometry data, and cytokines expression level were analyzed using unpaired two-tailed Student's $t$ test (when comparing two groups) or a one-way ANOVA with Tukey's post hoc test (when comparing $>2$ groups). Statistical analysis was performed by Student's two-tailed $t$ test; $p<0.05$ was considered statistically significant.

\section{Results}

RAM-589.555 diminishes disease severity in relapseremitting PLP 139-151 induced-EAE

RAM-589.555 treatment significantly reduced the severity of the extended EAE with decreased clinical signs as compared with the vehicle-treated group starting from day 11 through day $17(p<0.04)$ (Fig. 2a). RAM-589.555 not only delayed the second relapse but also decreased its severity. This RAM-589.555-induced alleviation was accompanied by a significant reduction of the maximal and cumulative clinical scores by 1.4-fold from $2.6 \pm 0.3$ and $19.5 \pm 4.3$ in vehicle-treated mice to $1.8 \pm 0.3$ and $10.7 \pm 1.9$, respectively, in RAM-589.555-treated mice (Table 1).

The histological analysis of spinal cord sections was on day 17 after treatment when clinical EAE score in RAM-589.555-treated mice was $0.2 \pm 0.1$ and in the vehicle animals the score was significantly higher $(1.4 \pm 0.1$; $p=0.04$ ).

RAM-589.555-treated mice had 10.0 times less inflammatory cellular infiltrates and widespread vacuoles
(Fig. 2b upper panel and 2c; $p=0.02$ ), 3.0 times less demyelinating areas (Fig. 2b middle panel and $1 \mathrm{~d} ; p=$ 0.007 ), and 8.6 times lower percent of axonal damaged areas (Fig. 2b, lower panel, Fig. 2e; $p$ 0.03) as compared to the vehicle-treated group.

RAM-589.555 accesses the CNS: pharmacokinetic analysis The mean concentration of RAM-589.555 was calculated for each sampling time point and a concentration curve was created (Fig. 3). RAM-589.555 was maximally absorbed in EAE mice $\left(T_{\max }\right)$ during the period between $1.5 \pm 0.3$ and $24 \mathrm{~h}$, with a mean maximal concentration $\left(C_{\max }\right)$ of $564 \pm 78$ and $251 \pm 0.7 \mathrm{ng} / \mathrm{ml}$ in blood and brain, respectively. Accordingly, the areas under the curve (AUC) were $8048 \pm 706.7$ and $4897 \pm 554.4 \mathrm{ng}$ "h/ $\mathrm{ml}$, respectively (Table 2). Considering the administered oral dose of $500 \mu \mathrm{g}$, RAM- 589.555 demonstrated $0.1 \%$ bioavailability in blood and a 2 -fold lower level in the CNS $(0.05 \%$ of the administered dose). These mean $T_{\max }, C_{\max }$, and AUCs of RAM-589.555 in EAE mice in blood and CNS were comparable with those of healthy mice (Additional file 4 and additional table 2).

\section{Effect of RAM-589.555 on viability and proliferation of primary microglia and astrocytes}

The concentrations of RAM-589.555 in the brain (90$250 \mathrm{ng} / \mathrm{ml}$ ) were then further investigated in the in-vitro experiments to evaluate its effects in microglia or astrocytes culture. Considering that the $C_{\max }$ of $250 \mathrm{ng} / \mathrm{ml}$ is equivalent to $400 \mathrm{nM}$, concentrations ranging between 25 and $400 \mathrm{nM}$ RAM-589.555 were chosen to assess its effect in-vitro.

Despite pre-rRNA transcript levels decreased with increasing RAM-589.555 concentrations (Fig. 4a), the viability and proliferation of microglia in response to LPS stimulation were not affected in the range of 25-200 nM and were only reduced at a concentration of $400 \mathrm{nM}$ RAM- 589.555 by $\sim 70 \%$ and $45 \%(p<0.05)$, respectively (Fig. 4b, c, left panel). Notably, viability and proliferation of astrocytes in response to TNFa activation were not affected by RAM-589.555 (Fig. 4b, c, right panel).

Nevertheless, incubation with RAM-589.555 did not modulate the level of BDNF (Fig. 5a), GDNF (Fig. 5b), and FGF2 (Fig. 5c).

\section{Effect of RAM-589.555 incubation on the morphology of primary microglia and astrocytes of naïve mice}

RAM-589.555 did not affect the morphology of activated microglia and astrocytes when compared with their control counterparts (Fig. 6). However, microglia density was slightly reduced at $400 \mathrm{nM}$ of RAM-589.555 accompanying the observed decrease in viability and proliferation. As shown by immunofluorescent staining, the shape of $\mathrm{CD}_{11 \mathrm{~b}^{+}}$microglia remained ramified and highly 

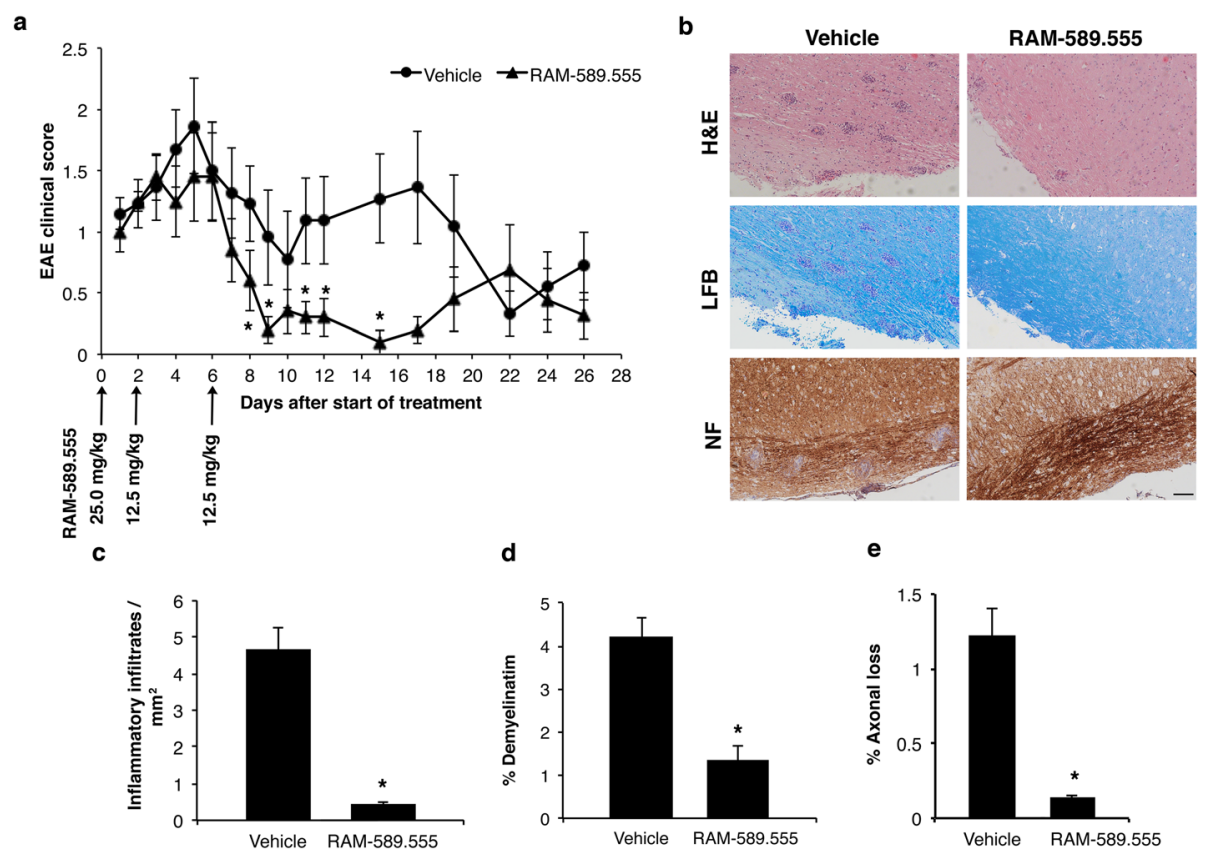

Fig. 2 RAM-589.555 treatment ameliorates EAE clinical signs and reduces pathological parameters. a EAE clinical score following treatment with RAM-589.555. $n=10$ mice/group; ${ }^{*} p<0.04$. Disease incidence 90\%. b Representative spinal cord sections (lumbar region) on day 17 after start of treatment stained with hematoxylin/eosin (H\&E, upper panel), luxol fast blue (LFB, middle panel), and neurofilament (NF, lower panel) in adjacent longitudinal spinal cord sections, day 17 post-RAM-589.555 treatment. Score of mice taken for IHC illustration: vehicle-treated mouse with score 3 compared with RAM-589.555-treated mouse with score 0 . Scale bar $=100 \mu \mathrm{m}, \mathbf{c}$ Mean number of inflammatory infiltrates per $\mathrm{mm}^{2}$ in adjacent longitudinal spinal cord sections. ${ }^{*} p=0.02$. $\mathbf{d}$ The percentage of demyelinating areas in adjacent longitudinal spinal cord sections. ${ }^{*} p=0.007$. e The percentage of axonal loss area in adjacent longitudinal spinal cord sections. ${ }^{*} p=0.03$. In $\mathbf{c}-\mathbf{e} n=3$ mice/group. The data presented are the mean \pm SEM according to three independent experimental repeats

branched with thin extensions and small round or oval somas at concentrations of 50, 100, and $400 \mathrm{nM}$ RAM589.555 , but had decreased density of $\mathrm{CD}_{11 b^{+}}$staining. The shape of $\mathrm{GFAP}^{+}$astrocytes was preserved and remained from polygonal to fusiform and flat morphology at all tested concentrations of RAM-589.555 (Fig. 6; The morphology of non-stimulated microglia and astrocytes is shown in Additional file 5a). High-power images show that although RAM-589.555 (400 $\mathrm{nM})$ reduces microglial viability, the morphology of remaining microglia is not hampered (Additional file 5b).

\section{Effect of RAM-589.555 on levels of pre-rRNA transcript in} blood cells, microglia, and astrocytes in-vivo

We have used the clinical profile described above (in Fig. 2a) to choose the time point for assessing the effect of RAM-589.555 on the in-vivo level of pre-rRNA transcript. Seven days after the first RAM-589.555 gavage, the level of pre-rRNA transcript in peripheral blood cells was significantly decreased by $38.6 \%(p<, 0.05$, compared to the vehicle-treated group, $n=6 /$ group) as shown in Fig. 7a. However, the pre-rRNA transcript level remained similar in microglia and astrocytes from RAM589.555-treated mice as compared to vehicle-treated mice (Fig. 7b, c).

\section{Identification of CNS-resident microglia and astrocytes using high-dimensional single-cell mass cytometry} High-dimensional characterization of astroglial and microglial populations in the CNS of healthy, vehicle-, and RAM-589.555-treated mice was performed on day 7 DPT (day post-treatment). RAM-589.555-treated mice sampled for mass cytometry showed a reduction in the clinical score by $87 \%$ as compared with

Table 1 EAE score following treatment with RAM-589.555

\begin{tabular}{lllll}
\hline Treatment & No. of mice & Peak of EAE (days after start of treatment) & Max clinical score & Cumulative disease score \\
\hline Vehicle & 10 & $3.4 \pm 0.6$ & $2.6 \pm 0.3$ & $19.5 \pm 4.3$ \\
RAM-589.555 & 10 & $2.3 \pm 0.7$ & $1.8 \pm 0.3$ & $10.7 \pm 1.9$ \\
${ }^{*} p$ value & & 0.09 & 0.04 & 0.03 \\
\hline
\end{tabular}

${ }^{*} T$ test 

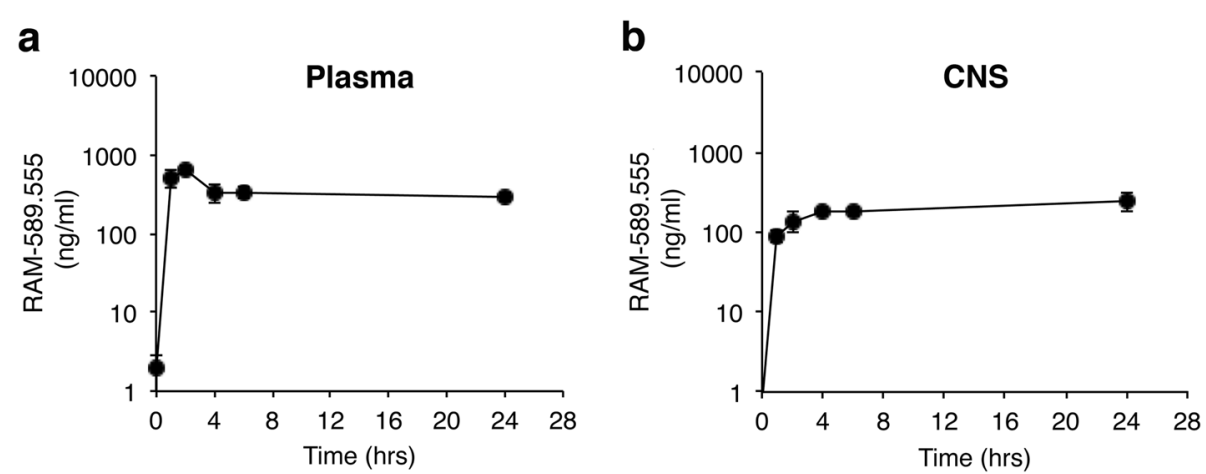

Fig. 3 RAM-589.555 accesses the CNS: pharmacokinetic analysis of RAM-589.555 in plasma and brain of EAE mice. The plasma (a) and CNS (b) concentration-time profiles of RAM-589.555 following $25 \mathrm{mg} / \mathrm{kg}$ oral dose in mice displaying EAE (results for pharmacokinetic analysis of RAM589.555 in healthy are shown in Additional file 4). Symbols indicate the observed plasma and CNS concentrations ( $n=4$ per time point). The data presented are the mean \pm SEM according to three independent experimental repeats

vehicle-treated mice (Fig. 8a). To exclude mistaken cell-sampling from circulating blood, we first perfused the mice and brains, and SCs were dissociated into single-cell suspensions so that samples included cells from the CNS parenchyma without the meninges (Additional file 2). To distinguish between resident microglia and infiltrating macrophages, we applied a strategy of analyzing CD45 expression, which is generally higher on infiltrating immune cells. The spade analysis enabled us to visually discriminate the CNSresident cells $\left(\mathrm{CD} 45^{\text {neg-low }}\right)$ from $\mathrm{CD} 45^{+}$infiltrating immune cells (CD45 high) (Fig. 8b).

Among the $\mathrm{CD} 45^{\text {neg-low }}$ cell population, we identified the $\mathrm{CD}_{11 \mathrm{~b}^{+}}$microglia and $\mathrm{GFAP}^{+}$astrocytes (Additional file 3). The ViSNE showed the different attributes of the population of $\mathrm{CD}_{4} 5^{+}$-infiltrating cells, $\mathrm{CD} 11 \mathrm{~b}^{+} \mathrm{CD} 45^{\text {low }}$ microglia, and $\mathrm{GFAP}^{+} \mathrm{CD} 45^{-}$astrocytes; all were increased in vehicle-treated mice displaying EAE (as compared with healthy mice), while moderately decreased in RAM-589.555-treated mice (Fig. 8c). Toxicity of RAM589.555 was assessed ex-vivo by measuring the viability based on Rh gating suggesting that viability of astrocytes and microglia was not hampered in RAM-589.555treated mice (Additional file 6).

The spade analysis showed clusters of astrocytes $\left(\mathrm{GFAP}^{+} \mathrm{CD}^{-} 5^{-}\right.$; Fig. 9a left panel) that seemingly are activated astrocytes based on GFAP expression and microglia $\left(\mathrm{CD} 11 \mathrm{~b}^{+} \mathrm{CD} 45^{\text {low }}\right.$; Fig. 9a, right panel) across all tested groups. Interestingly, the number of astrocytes

Table 2 Pharmacokinetic features of RAM-589.555 in blood and CNS of mice displaying EAE

\begin{tabular}{lll}
\hline $\mathbf{2 5} \mathbf{~ m g} / \mathbf{k g}$ oral gavage & CNS & Plasma \\
\hline Cmax ng/ml & $251 \pm 0.7$ & $564 \pm 78$ \\
Tmax, h & 24 & $1.5 \pm 0.3$ \\
AUC $_{0-t,}, \mathrm{ng}$ *h/ml & $4897 \pm 554.4$ & $8048 \pm 706.7$ \\
\hline
\end{tabular}

and microglia was increased in EAE mice as compared with naive mice and decreased in RAM.589.555-treated mice (as presented in Fig. 9a in boxes of left and right panels, respectively). Quantitative analysis showed that percentages of astrocytes and microglia were significantly increased in vehicle-treated mice by $34 \%$ and $75 \%$ (1.5- and 4-fold, respectively, as compared with control; $p<0.01)$. Notably, these percentages decreased in RAM589.555 -treated mice by $31 \%$ and $59 \%$, respectively (1.4and 2.4-fold as compared with vehicle-treated mice; $p<$ 0.05 for microglia and $p<0.01$ for astrocytes), suggesting that RAM-589.555 decreases neuroinflammation (Fig. 9b, c, respectively; an additional staining of F4/80 was used to label microglia in Additional file 7a). Notably, the proportion of microglia expressing CX3CR1 was decreased in vehicle-treated mice by $25 \%(p<0.05)$ but then was not significantly recovered in RAM589.555-treated mice (Additional file 7b-c).

High-dimensional single-cell mass cytometry of CNSresident microglia and astrocytes reveals the beneficial role of RAM-589.555

Analysis of cytokines in astrocytes showed increased expression of pro-inflammatory cytokines, IL-1b, IL-6, IL-12, IL-17, TNF $\alpha$, and IFN $\gamma(p<0.05)$ in EAE-mice that significantly decreased by $31-51 \%$ after RAM589.555 treatment compared to vehicle treatment $(p<$ 0.05; Fig. 10a). The levels of anti-inflammatory cytokines IL-4, IL-10, and TGF $\beta$ of astrocytes were decreased in EAE-mice and significantly increased by $31-42 \%$ in RAM-589.555-treated mice ( $p<0.05$, compared to vehicle-treated mice; Fig. 10b). These results suggest that RAM-589.555 could shift astrocytes to anti-inflammatory phenotype. Interestingly, this was accompanied with decreased number of infiltrating $\mathrm{CD}^{+} \mathrm{T}$ cells (Additional file 8a) with decrease in pro-inflammatory cytokines (Additional file $8 \mathrm{~b}$ ) and 

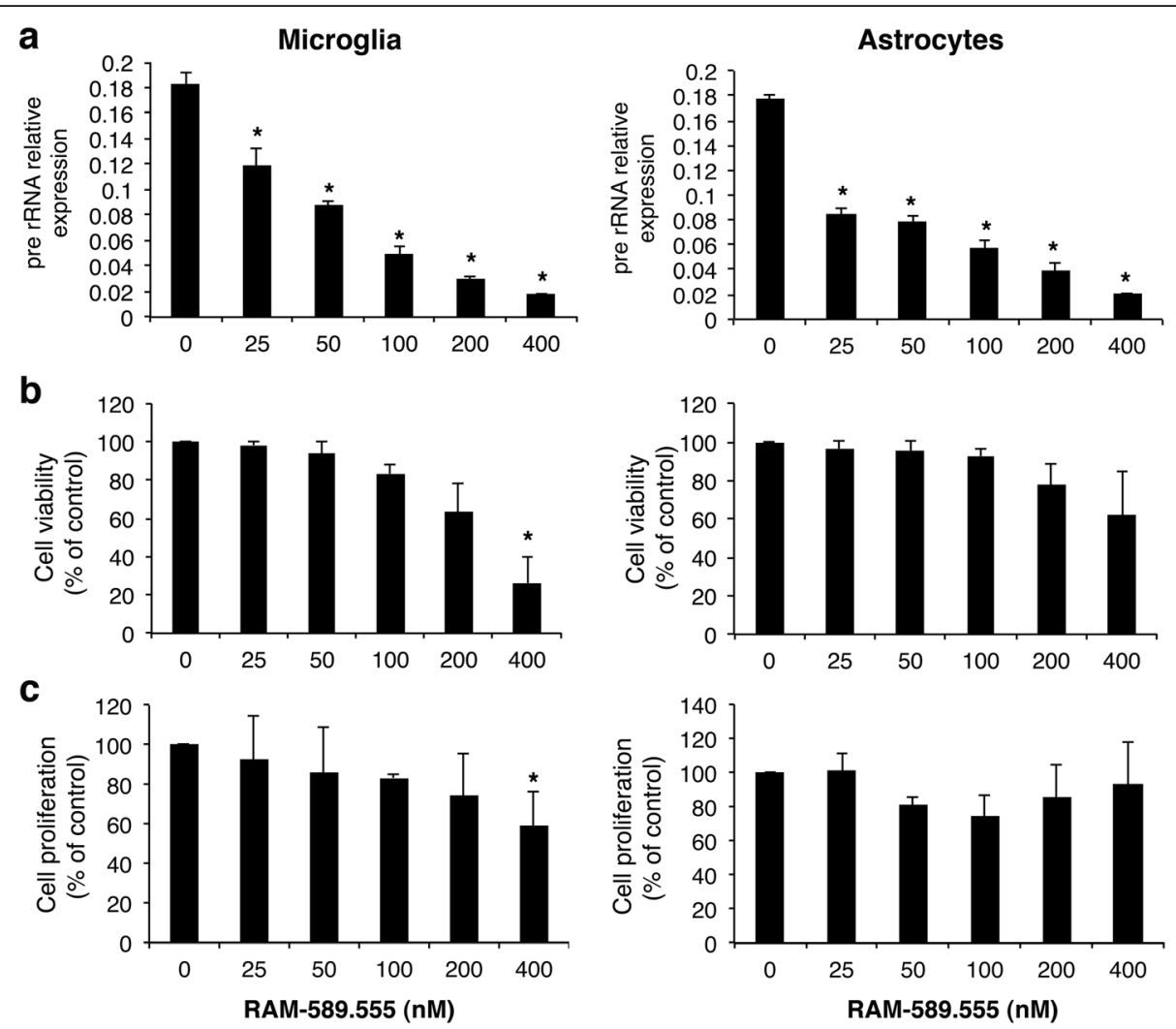

Fig. 4 Effect of RAM-589.555 on pre-rRNA expression, viability, and proliferation of microglia and astrocytes. a-c LPS-activated microglia and TNFa-activated astrocytes were cultured in the absence or presence of RAM-589.555 (25-400 nM) for $48 \mathrm{~h}$. $\mathbf{a}$ Level of pre-rRNA transcript, $\mathbf{b}$ viability, and c proliferation of microglia and astrocytes were analyzed following exposure to RAM-589.555. In (b) and (c), results are presented as the percentage relative to control untreated cells. ${ }^{*} p<0.05$ vs. control. Error bars represent mean \pm SEM according to three independent experimental repeats $(n=3$ in each experiment)

increase in anti-inflammatory cytokines (Additional file 8c), suggesting that RAM-589.555 has a direct effect at least on $\mathrm{CD} 4^{+}$-lymphocytes and their phenotype.

Similar to astrocytes, the whole $\mathrm{CD}_{11 \mathrm{~b}^{+}}$microglia population showed increased expression of proinflammatory cytokines IL-1b, IL-6, IL-12, IL-17, TNF $\alpha$, and IFN $\gamma$ in EAE-mice, which was significantly decreased by $33-77 \%$ in RAM-589.555-treated mice. On the contrary, the levels of anti-inflammatory cytokines IL-10 and TGF $\beta$ were decreased in EAE-mice and significantly increased by $38-49 \%$ in RAM-589.555-treated mice (Fig. 10c, d).

\section{Discussion}

Our results show that RAM-589.555 accesses the CNS of mice displaying EAE, reduces the number of infiltrating immune cells, and thereby ameliorates EAE. Clinically relevant concentrations of RAM- 589.555 not only decrease the number of microglia and astrocytes in-vivo but further favors their anti-inflammatory cytokine profile as demonstrated by high-dimensional single-cell mass cytometry. The observed restoration of neuroprotective potential of CNS-resident microglia and astrocytes could explain the amelioration of relapsingremitting EAE and decreased CNS-inflammation under RAM-589.555 treatment. In-vitro, the viability, proliferation, and NFs secretion capacity of astrocytes were not affected by RAM-589.555; however, microglia were sensitive and their viability and proliferation were decreased, by 400 nM RAM- 589.555 without affecting their NFs secretion.

Nevertheless, the in-vivo results show comparable sensitivity of microglia and astrocytes to RAM-589.555. This putative inconsistence with the in-vitro results probably results from the stimulus used for each culture, the assay used to measure cell proliferation, or with both. Apparently, the in-vitro conditions are far from being similar to the in-vivo scenario.

As compared to the high RAM-589.555 sensitivity of splenocytes and PBMC described in our previous studies [19] at the range of $25-400 \mathrm{nM}$, astrocytes are distinctly resistant and microglia are mildly responsive to RAM589.555 in-vitro; furthermore, their populations are even restored in-vivo. These distinct in-vitro sensitivity 
a

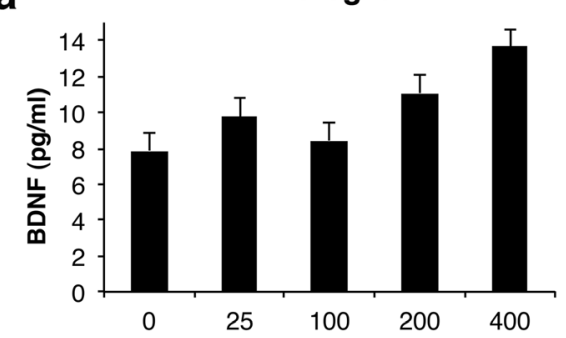

b

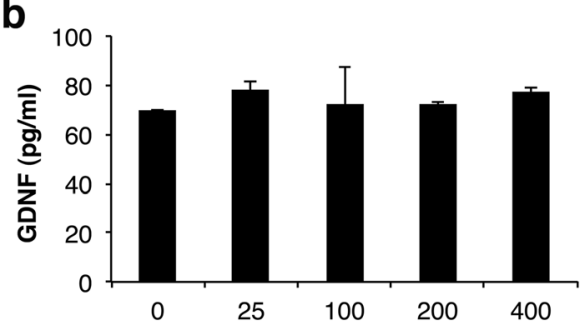

C

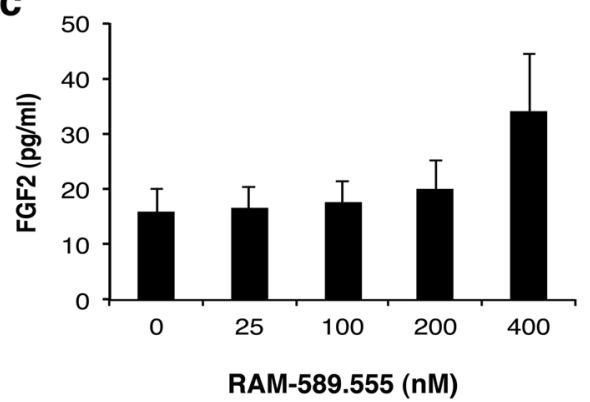

Astrocytes
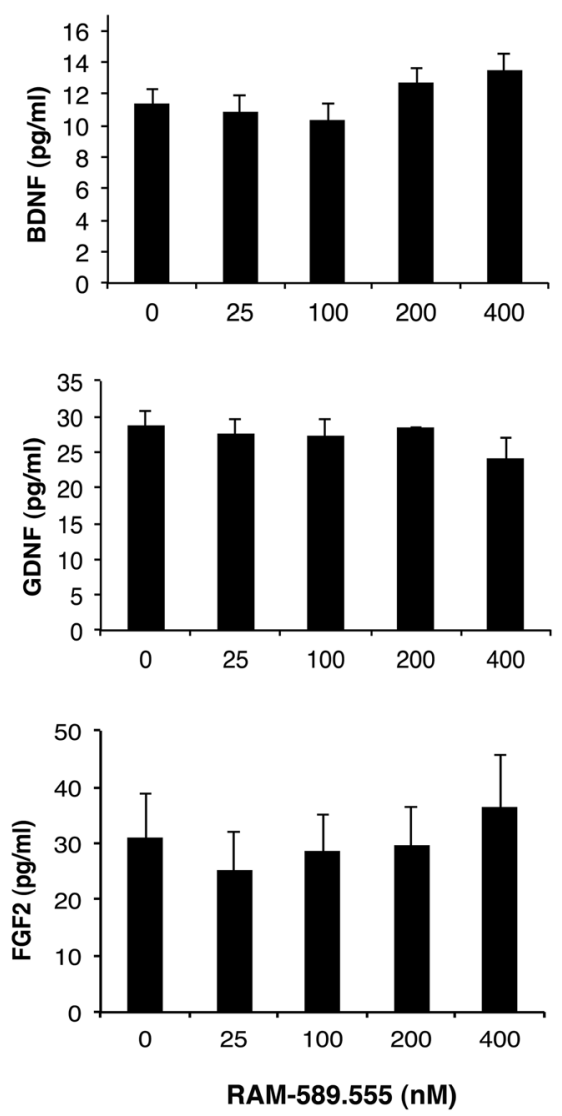

Fig. 5 RAM-589.555 does not modulate the secretion of BDNF, GDNF, and FGF2 from activated microglia and astrocytes. LPS-activated microglia and TNFa-activated astrocytes were cultured in the absence or presence of RAM589.555 at 25-400 nM for 48 h. Levels of BDNF (a), GDNF (b), and FGF2 (c) were measured in supernatants by ELISA. Error bars represent \pm SEM according to three independent experimental repeats (duplicate/experiment)

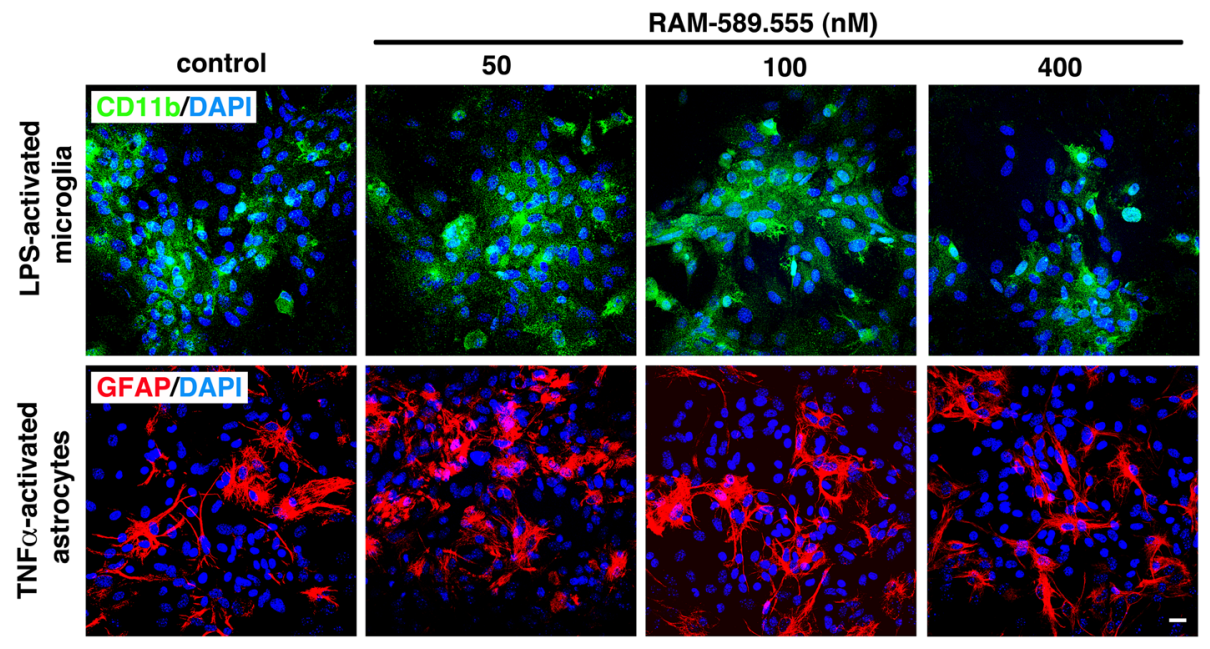

Fig. 6 RAM-589.555 does not modulate the morphological shaping of activated microglia and astrocytes. LPS-activated microglia and TNFaactivated astrocytes were cultured in the absence or presence of RAM-589.555 at 50 and $100 \mathrm{nM}$ for $48 \mathrm{~h}$. a Bright field phase contrast images show microglia (upper panel) and astrocytes (lower panel). Scale bar $=50 \mu \mathrm{m}$. b Immunofluorescence microscopy images show CD11 ${ }^{+}$ microglia and GFAP ${ }^{+}$astrocytes stained with DAPI as a marker for cell viability. Images are representative of three independent experimental repeats (4 images per condition). Scale bar $=20 \mu \mathrm{m}$ 

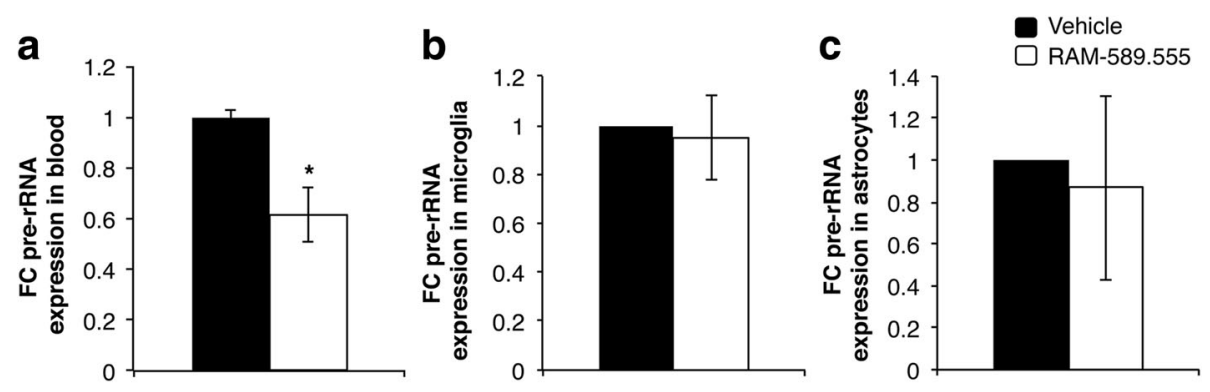

Fig. 7 Effect of RAM-589.555 in-vivo on pre-rRNA transcript level in blood cells (a) and CNS's resident glial cells; microglia (b) and astrocytes (c). On the day of disease onset (score 1), mice received RAM-589.555 by oral gavage. Mice were bled at day 7 after initial RAM-589.555 gavage as described in methods into heparinized tube; cells were lysed and further processed for qRT-PCR. Presented as fold change (FC) compare prerRNA transcript in healthy mice ( $n=6$ mice/group). The data presented are the mean \pm SEM according to three independent experimental repeats. ${ }^{*} p<0.05$ in RAM-589.555 vs. vehicle
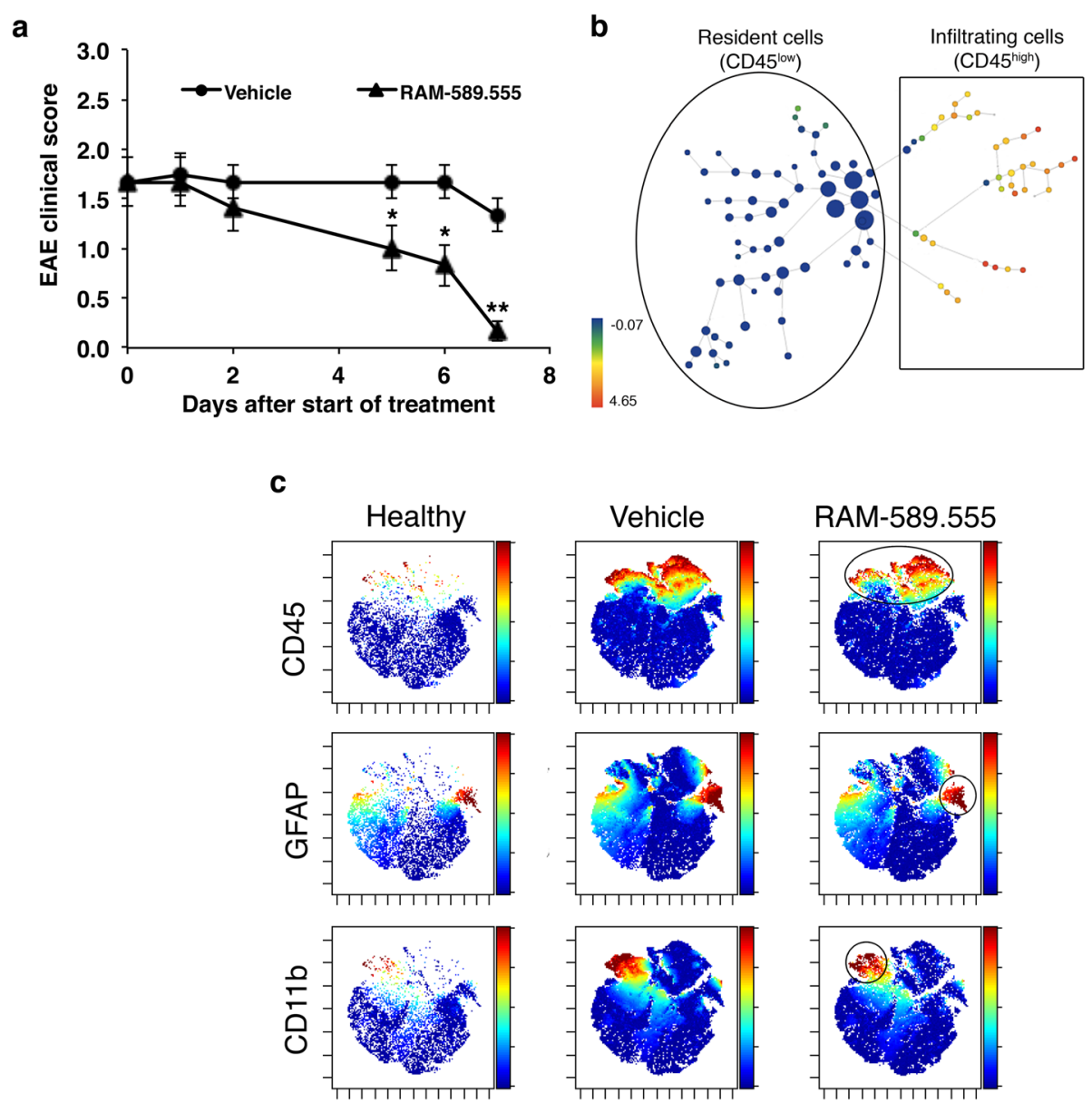

RAM-589.555

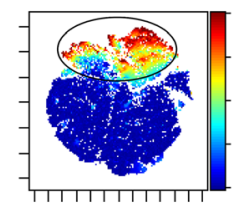

Fig. 8 Characterization of the CNS's resident astroglial and microglial cell populations by mass cytometry. a EAE clinical score of mice subjected for mass cytometry following treatment with RAM-589.555 (sampling at day 7 after start of RAM-589.555 treatment). $n=6$ mice/group; ${ }^{*} p<0.04$, ${ }^{* *} p<0.01$ vs. vehicle-group. Disease incidence is $90 \%$. b Spade diagram visualizing CD45 ${ }^{\text {low }}$ CNS residents and CD45 high infiltrating cells. c ViSNE diagrams of brain resident cells visualize the clusters under RAM-589.555 as compared with vehicle-treated and healthy mice. The color code shows the expression levels of CD45, GFAP, and CD11b. Representative diagrams are shown ( $n=6$ mice/group). The location of the marker positive cells is shown by the ellipse or circle. The data presented are the mean \pm SEM according to three independent experimental repeats 

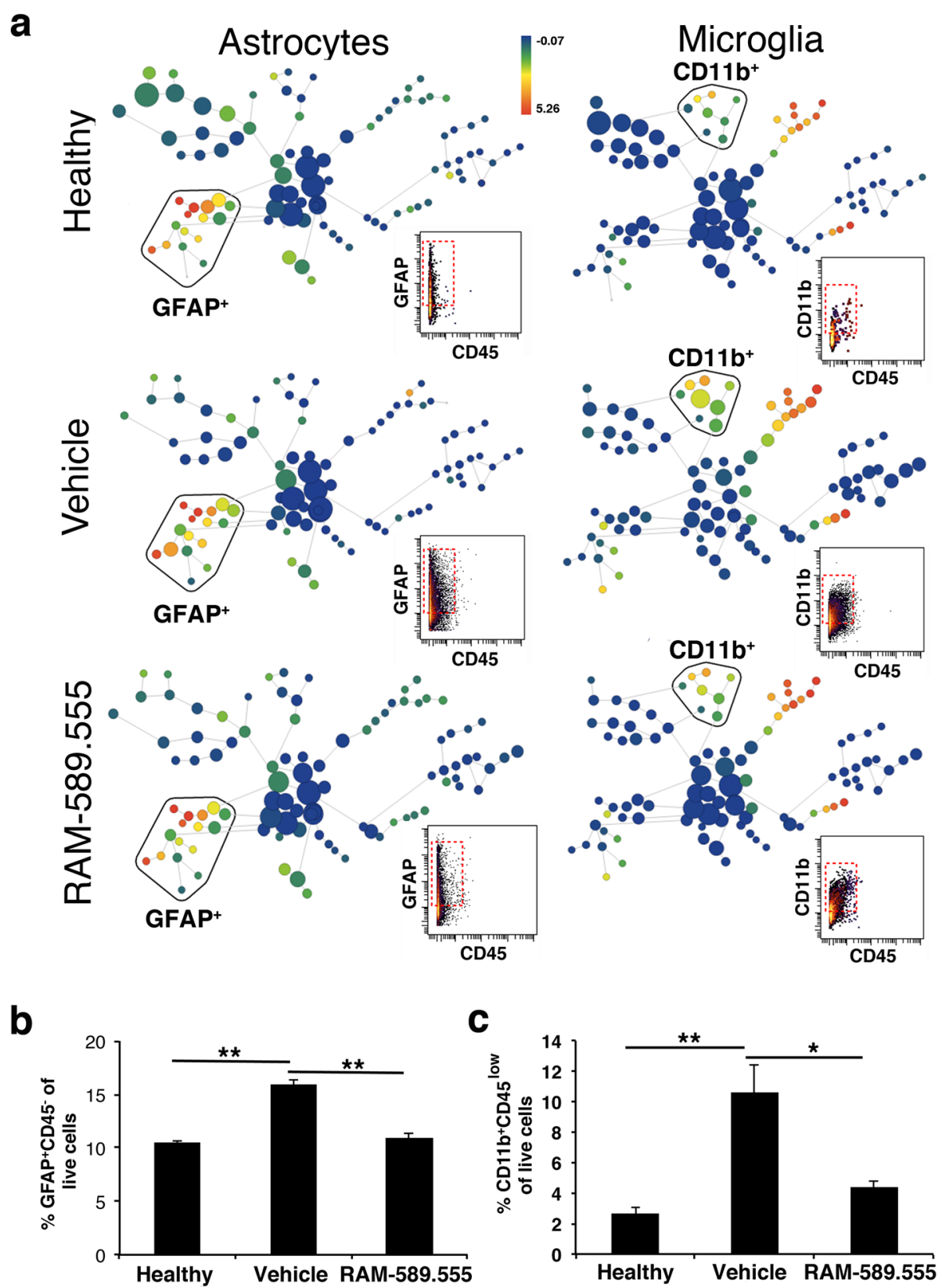

Fig. 9 Differential abundance of astroglial and microglial cell populations in RAM-589.555-treated mice. a Spade diagrams of brain resident cells visualize the clusters under the RAM-589.555 as compared with vehicle-treated and healthy mice. The color code shows the expression levels of $\mathrm{GFAP}^{+}$and $\mathrm{CD} 11 \mathrm{~b}^{+}$cell populations. Representative diagram are shown ( $n=6$ mice/group). Dot plots demonstrate the marker labeling on live single cells. GFAP ${ }^{+} \mathrm{CD} 45^{-}$and $\mathrm{CD} 11 \mathrm{~b}^{+} \mathrm{CD} 45^{\text {low }}$ populations are contoured by red dashed rectangle. $\mathbf{b}$, $\mathbf{c}$ Frequencies of CNS-resident astroglial and microglial cells in RAM-589.555-treated mice as compared with vehicle-treated and healthy mice. The identified astroglia and microglial populations (b and $\mathbf{c}$, respectively) and relative abundance are presented ( $n=6$ in each group). The data presented are the mean \pm SEM according to three independent experimental repeats ${ }^{*} p<0.05 ;{ }^{* *} p<0.01$. Statistical significance was determined by one-way ANOVA with post-hoc Tukey test

profiles can be attributed to higher proliferation activity of PHA-stimulated PBMC, whereas stimulation of astrocytes and microglia by TNFa and LPS, respectively, induces activation rather than proliferation.

The differential responsiveness of PBMC and microand astroglial cells was also evidenced by a difference in level of pre-rRNA transcript in-vivo suggesting an ontarget activity of RAM-589.555. Yet, the possibility of minor off-target activity cannot be excluded. In-vivo, this differential responsiveness can also be attributed to a lower concentration of RAM-589.555 measured in CNS as compared to blood, as well as to a relatively higher 

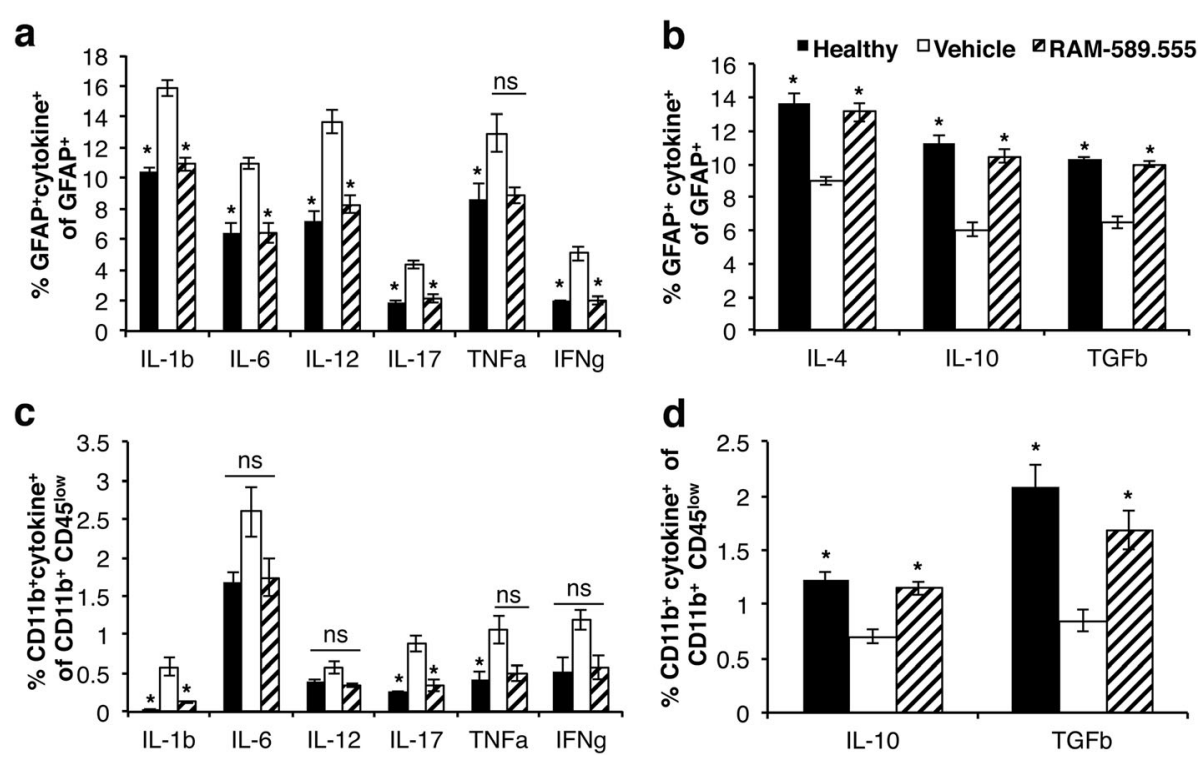

Fig. 10 Expression of pro/anti-inflammatory cytokines following RAM-589.555 treatment. $\mathbf{a}$, $\mathbf{b}$ Expression of pro/anti-inflammatory cytokines by astrocytes and $\mathbf{c}$, d microglia. ( $n=6$ in each group) The data presented are the mean \pm SEM according to three independent experimental repeats. ${ }^{*} p<0.05$ vs. vehicle; $n s$ not significant. Statistical significance was determined by one-way ANOVA with post-hoc Tukey test

proliferation rate of pathogenic immune cells as compared to CNS-resident glial cells.

Interestingly, although RAM-589.555 does not affect proliferation, viability (except for $400 \mathrm{nM}$ in microglia culture), and secretion of neurotrophic factors, its capacity to modulate the proportions of micro- and astroglial cells and its differential effect on their level of pre-rRNA transcript, as compared with PBMC from EAE-mice, suggests the possibility that RAM-589.555 exerts an indirect effect on micro- and astroglial cells rather than a direct effect. Alternatively, as RAM-589.555 crosses into the CNS of healthy and EAE mice, it may have non-POL1-mediated effects on microglia and astrocytes within the CNS, which warrants further investigation. Yet, we cannot exclude that this difference could be due to lower concentrations of the drug in the CNS.

Indeed, the pharmacokinetic findings suggest that RAM-589.555 can access both the blood and CNS after oral gavage, which is comparable with our previous studies showing the permeability characteristics of RAM589.555 in the CACO-2 model with a medium-high rate of permeability [19]. The comparable pharmacokinetic parameters of RAM-589-555 in healthy and EAE mice suggest the active penetrance of RAM- 589.555 through the $\mathrm{BBB}$ rather than passive entry of RAM-589.555 through the permeabilized BBB of EAE mice. Moreover, considering that the permeabilized $\mathrm{BBB}$ is characteristic of neuroinflammation in EAE-affected mice, one would anticipate RAM-589.555 in the CNS to be 2-fold lower than in blood. Therefore, the presence of 250 and 290 $\mathrm{ng} / \mathrm{ml}$ of RAM-589.555 in CNS and blood, respectively,
$24 \mathrm{~h}$ after a single dose of $25 \mathrm{mg} / \mathrm{kg}$ suggests slow clearance rate that should be evaluated in further study. Our findings suggest that RAM-589.555 does not harm and even preserves the functionality of microglia and astrocytes in regards to the neuroprotective potential of glial cells.

It has been shown that mice lacking CX3CR1 are more susceptible to EAE, with a significantly earlier onset and higher incidence of the disease [30]. This, together with conclusions from bone marrow chimeras that CX3CR1 regulates myeloid and possibly microglial cell responses [31, 32], suggests that CX3CR1 would act to dampen neuroinflammation. This supports our data showing that proportions of microglia expressing CX3CR1 are decreased in vehicle-treated mice; however, these microglia expressing CX3CR1 do not recover in RAM-589.555treated mice. Despite the evidences of a neuroprotective role for CX3CR1 and its ligand fractalkine, there are data suggesting that this receptor-ligand axis is detrimental in some settings [33].

Although we cannot point to a classical neurotoxic A1/neuroprotective A2 characterization of the astrocyte population, the dynamic expression of pro- and antiinflammatory cytokines demonstrated herein, and previous characterization of two distinct subpopulations [34], suggests that RAM-589.555 induces a shift toward an anti-inflammatory cytokine profile that favors the neuroprotective functions of astrocytes.

It has been suggested that microglial activation is necessary for IL-1 $\beta$-mediated astrocyte activation [7]; although we could not conclude it from our data herein, 
the increased expression of IL-1 $\beta$ and IL-17 by microglia (also shown in [35]) may suggest that while it activates astrocytes during EAE progression, there is a reversal effect following RAM-589.555 treatment.

In accordance, we also found that pro-inflammatory IL-1 $\beta$, IL-17 were produced by astrocytes. Because microglia also produce IL-1 $\beta$ and IL-17, these cytokines may act in an paracrine manner to induce IL-17 expression in astrocytes, and thereby contribute to autoimmune diseases [36]. Our data show that about $2 \%$ of astrocytes in healthy mice secrete IL-17; this finding is corroborated by recent study showing the neuronal protection effect of IL-17 [37]. Thus, like IL- 6 and TGF $\beta$ that produced by reactive astrocytes, IL-17 can play both as key component and as neurotrophic factor in neuroinflammation.

Indeed, our results show the presence of IL-6- and TGF $\beta$-secreting astrocytes and microglia in the CNS of healthy mice. In view of previous studies demonstrating the pleiotropic functions of these factors under homeostasis and demyelinating diseases [8, 38-40] and their opposite direction in RAM-589.555-treated mice as compared with vehicle-treated mice, it is likely that IL-6 and TGF $\beta$ secreted by astrocytes play a protective rather a detrimental role.

The level of IFNY was decreased in astrocytes from RAM-589.555-treated mice, which is in line with observations that IFN $\gamma$ can potentiate reactive astrogliosis, suppress remyelination, and delay disease recovery [41]. The notion that RAM-589.555 ameliorated clinical severity and also decreased the astrocytic IL- 6 expression is in line with previous studies implicating astrocyte-secreted IL-6 in EAE pathogenesis $[38,42]$. The increase of TGF $\beta^{+}$-astrocytes in RAM-589.555-treated mice corroborates demonstrations from previous studies that $\mathrm{TGF} \beta^{+}$-astrocytes exhibit beneficial effects by limiting immune cell infiltration after stroke [43] and by secreting fundamental antiinflammatory cytokines, IL-4 and IL-10, which decrease proliferation and activation of infiltrating immune cells and support remyelination by promoting oligodendrocyte progenitor maturation [44]. In summary, RAM-589.555 accesses the CNS, reduces the number of infiltrating immune cells, and of microglia and astrocytes while maintaining their anti-inflammatory cytokine profile. These findings suggest that the therapeutic mechanism underlying the amelioration of relapsing-remitting EAE by RAM-589.555 is associated with decreased CNS inflammation and, most importantly, restoration of the neuroprotective and anti-inflammatory capacity of CNSresident microglia and astrocytes.

\section{Conclusions}

This study highlights the correlation of the therapeutic potential of the CNS-permeable POL1 inhibitor RAM-
589.555 in MS-like disease with likely indirect antiinflammatory and neuroprotective effects in CNSresident microglia and astrocytes. Whether mediated by its effect on the infiltrating immune cells or these of the peripheral circulating blood, warrants further investigation. Notably, the accessibility of RAM-589.555 to the CNS and its beneficial effect on microglia and astrocytes raises the possibility of potential beneficial maintaining effect on other CNS-resident cells, neurons and oligodendrocytes, which warrants further investigation.

\section{Supplementary information}

Supplementary information accompanies this paper at https://doi.org/10. 1186/s12974-020-01983-2.

Additional file 1. Schematic representation of the experimental procedure for the characterization of the brain's resident and infiltrating immune cell population by mass cytometry.

Additional file 2. Evaluation of perfusion quality. Identification of CD45 cells in the CNS-compartment that were present in the blood circulation at the time of mouse perfusion (perfusion validation). Blue arrows show the analysis that was done and black arrows show representative results.

Additional file 3. Gating strategy for mass cytometry single-cell analysis. The single-cell analysis was performed on live single cells, chosen by the following gating strategy: cell length versus Iridium (Ir) 191/193 (choosing singlets), Ir191 versus Ir193 (choosing live cells), and Ir191/193 versus Rhodium (Rh; excluding dead cells). CD11 $\mathrm{b}^{+}$microglia and GFAP ${ }^{+}$astrocytes were picked from CD45 $5^{\text {negative-low }}$ (CD45 $5^{\text {neg-low }}$ ) population. Representative image of six repeats.

Additional file 4:. Pharmacokinetic analysis of RAM-589.555 in plasma and brain of healthy mice. The plasma (a) and CNS (b) concentrationtime profiles of RAM-589.555 following $25 \mathrm{mg} / \mathrm{kg}$ oral dose in agematched healthy mice. Symbols indicate the observed plasma and CNS concentrations ( $n=4$ per time point). The data presented are the mean \pm SEM according to three independent experimental repeats.

Additional file 5. Morphology of microglia and astrocytes. a. Morphology of non-stimulated microglia and astrocytes. b. High-power images show that although RAM-589.555 (400 nM) reduces microglial viability, the morphology of remaining microglia is not hampered. Left bar= $20 \mu \mathrm{m}$; right bar $=10 \mu \mathrm{m}$.

Additional file 6 . The proportion of live microglia and astrocytes in mice treated with RAM-589.555 as compared with Vehicle-treated mice and healthy mice. Percentages of live microglia (CD11 b ${ }^{+}$CD $\left.45^{\text {low }}\right)$ and astrocytes $\left(\mathrm{GFAP}^{+} \mathrm{CD} 45^{\circ}\right)$ in the CNS compartment based on Rh gating (percentages are shown). Representative results of 6 repeats are shown.

Additional file 7: Analysis of microglia. a. Frequency of $\mathrm{F} 4 / 80^{+} \mathrm{CD} 45^{\mathrm{low}}$ microglia in RAM-589.555-treated mice as compared with vehicle-treated mice and healthy mice. b. The proportions of $C D 11 b^{+} C D 45^{\text {low }}$ microglia expressing CX3CR1. Shown are representative results of three independent repeats. C. Quantitative analysis of microglia (CD11 b $\left.\mathrm{CD}^{+} 5^{\text {low }}\right)$ expressing CX3CR 1 in the CNS compartment. $n=6$ in each group. The data presented are the mean \pm SEM according to three independent experimental repeats ${ }^{*} p<0.05$. Statistical significance was determined by oneway ANOVA with post-hoc Tukey test.

Additional file 8: Expression of pro/anti-inflammatory cytokines by CNSinfiltrating $\mathrm{CD}^{+}{ }^{\mathrm{T}}$ cells following RAM-589.555 treatment in EAE mice. a. Frequency of CNS-infiltrating CD4 ${ }^{+} \mathrm{T}$ cells in RAM-589.555-treated mice as compared with vehicle-treated and healthy mice. The identified CD4 ${ }^{+}$ $T$ cells and relative abundance is presented ( $n=6$ in each group). b-c Expression of pro/anti-inflammatory cytokines by $C D 4^{+} T$ cells. ( $n=6$ in each group) The data presented are the mean \pm SEM according to three independent experimental repeats. ${ }^{*} p<0.05$ vs. vehicle. Statistical significance was determined by one-way ANOVA with post-hoc Tukey test. 
Additional file 9: Table 1. CyTOF mass cytometry antibody panel. The full metal-conjugated antibody panel used for CyTOF mass cytometry experiments.

Additional file 10: Table 2. Pharmacokinetic features of RAM-589.555 in blood and CNS of healthy mice.

\section{Abbreviations}

MS: Multiple sclerosis; CNS: Central nervous system; POL1: RNA polymerase 1; EAE: experimental autoimmune encephalomyelitis; BBB: Blood-brain barrier: P3: Postnatal day 3; DMEM: Dulbecco's modified Eagle's medium; PDL: PolyD-lysine; BDNF: Brain-derived neurotrophic factor; GDNF: Glial-derived neurotrophic factor; SCs: Spinal cords; DPT: Days post treatment; HE: Hematoxylin and eosin; LFB: Luxol fast blue; NF: Neurofilament; SPADE: Spanning-tree progression analysis of density-normalized events; ViSNE: t-distributed stochastic neighbor embedding (t-SNE)-based visualization

\section{Acknowledgements}

We thank A. Grau from the Biomedical core facility at the Technion Faculty of Medicine for technical support and comments.

\section{Authors' contributions}

$R Z F, M G$, and AA conceived the study and designed the experiments. RZF, $T R Z$, and $L C$ performed the experiments. RZF, MG, and AA wrote the manuscript. All authors discussed the results and conclusions and reviewed the manuscript. All authors read and approved the final manuscript.

\section{Funding}

Not applicable

\section{Availability of data and materials}

The data supporting the conclusions of this article are included within the article and its additional files.

\section{Ethics Approval and Consent to participate}

All procedures involving animals were conducted in in compliance with the Israeli Council for Animal Care guidelines.

\section{Consent for publication}

Not applicable.

\section{Competing interests}

The authors declare that they have no competing interests.

\section{Author details}

${ }^{1}$ Neuroimmunology Laboratory, Multiple Sclerosis Center, Sheba Medical Center, Ramat Gan, Israel. ${ }^{2}$ Sackler School of Medicine, Tel-Aviv University, Tel Aviv, Israel.

\section{Received: 6 February 2020 Accepted: 5 October 2020}

Published online: 21 October 2020

\section{References}

1. Achiron A, Polliack M, Rao SM, Barak Y, Lavie M, Appelboim N, Harel Y. Cognitive patterns and progression in multiple sclerosis: construction and validation of percentile curves. J Neurol Neurosurg Psychiatry. 2005;76:744-9.

2. Compston A, Coles A. Multiple sclerosis. Lancet. 2008;372:1502-17.

3. Confavreux $C$, Vukusic $S$. The clinical epidemiology of multiple sclerosis. Neuroimaging Clin N Am. 2008;18:589-622 ix-x.

4. Gandhi R, Laroni A, Weiner HL. Role of the innate immune system in the pathogenesis of multiple sclerosis. J Neuroimmunol. 2010;221:7-14.

5. Weiner HL. A shift from adaptive to innate immunity: a potential mechanism of disease progression in multiple sclerosis. J Neurol. 2008;255(Suppl 1):3-11.

6. Monney L, Sabatos CA, Gaglia JL, Ryu A, Waldner H, Chernova T, Manning S, Greenfield EA, Coyle AJ, Sobel RA, et al. Th1-specific cell surface protein Tim-3 regulates macrophage activation and severity of an autoimmune disease. Nature. 2002:415:536-41.

7. Liddelow SA, Guttenplan KA, Clarke LE, Bennett FC, Bohlen CJ, Schirmer L, Bennett ML, Munch AE, Chung WS, Peterson TC, et al. Neurotoxic reactive astrocytes are induced by activated microglia. Nature. 2017;541:481-7.
8. Traiffort E, Kassoussi A, Zahaf A, Laouarem Y. Astrocytes and microglia as major players of myelin production in normal and pathological conditions. Front Cell Neurosci. 2020;14:79.

9. Clemente D, Ortega MC, Melero-Jerez C, de Castro F. The effect of glia-glia interactions on oligodendrocyte precursor cell biology during development and in demyelinating diseases. Front Cell Neurosci. 2013;7:268.

10. Domingues HS, Portugal CC, Socodato R, Relvas JB. Oligodendrocyte, astrocyte, and microglia crosstalk in myelin development, damage, and repair. Front Cell Dev Biol. 2016;4:71.

11. Lloyd AF, Davies CL, Miron VE. Microglia: origins, homeostasis, and roles in myelin repair. Curr Opin Neurobiol. 2017;47:113-20.

12. Armstrong RC. Growth factor regulation of remyelination: behind the growing interest in endogenous cell repair of the CNS. Future Neurol. 2007;2:689-97.

13. Ku MC, Wolf SA, Respondek D, Matyash V, Pohlmann A, Waiczies S, Waiczies H, Niendorf T, Synowitz M, Glass R, Kettenmann H. GDNF mediates glioblastoma-induced microglia attraction but not astrogliosis. Acta Neuropathol. 2013:125:609-20

14. Patil SP, Liu C, Alban J, Yang N, Li XM. Glycyrrhiza uralensis flavonoids inhibit brain microglial cell TNF-a secretion, p-lkB expression, and increase brainderived neurotropic factor (BDNF) secretion. Journal of Traditional Chinese Medical Sciences. 2014;1:28-37.

15. Baecher-Allan C, Kaskow BJ, Weiner HL. Multiple sclerosis: mechanisms and immunotherapy. Neuron. 2018:97:742-68.

16. Achiron A, Feldman A, Magalashvili D, Dolev M, Gurevich M. Suppressed RNA-polymerase 1 pathway is associated with benign multiple sclerosis. PLoS One. 2012;7:e46871.

17. Zilkha-Falb R, Gurevich M, Achiron A. Experimental autoimmune encephalomyelitis ameliorated by passive transfer of polymerase 1-silenced MOG35-55 lymphatic node cells: verification of a novel therapeutic approach in multiple sclerosis. NeuroMolecular Med. 2017;19:406-12.

18. Achiron A, Mashiach R, Zilkha-Falb R, Meijler MM, Gurevich M. Polymerase I pathway inhibitor ameliorates experimental autoimmune encephalomyelitis. J Neuroimmunol. 2013;263:91-7.

19. Achiron A, Zilkha-Falb R, Mashiach R, Gurevich M. RAM-589.555 a new Polymerase-1 inhibitor as innovative targeted-treatment for multiple sclerosis. J Neuroimmunol. 2017:302:41-8.

20. Feldmann M, Pathipati P, Sheldon RA, Jiang X, Ferriero DM. Isolating astrocytes and neurons sequentially from postnatal murine brains with a magnetic cell separation technique, vol. 2014; 2014.

21. Harms AS, Tansey MG. Isolation of murine postnatal brain microglia for phenotypic characterization using magnetic cell separation technology. Methods Mol Biol. 2013;1041:33-9.

22. Fisher J, Levkovitch-Verbin H, Schori H, Yoles E, Butovsky O, Kaye JF, BenNun A, Schwartz M. Vaccination for neuroprotection in the mouse optic nerve: implications for optic neuropathies. J Neurosci. 2001;21:136-42.

23. Allgoewer A, Schmid M, Radermacher P, Asfar P, Mayer B. Area under the curve-derived measures characterizing longitudinal patient responses for given thresholds. Epidemiol Biostat Public Health. 2018;15(4):e12948-1 e12948-11.

24. Zilkha-Falb R, Gurevich M, Hanael E, Achiron A. Prickle1 as positive regulator of oligodendrocyte differentiation. Neuroscience. 2017;364:107-21.

25. Mrdjen D, Hartmann FJ, Becher B. High Dimensional cytometry of central nervous system leukocytes during neuroinflammation. Methods Mol Biol. 2017;1559:321-32.

26. Korin B, Ben-Shaanan TL, Schiller M, Dubovik T, Azulay-Debby H, Boshnak NT, Koren T, Rolls A. High-dimensional, single-cell characterization of the brain's immune compartment. Nat Neurosci. 2017;20:1300-9.

27. Korin B, Dubovik T, Rolls A. Mass cytometry analysis of immune cells in the brain. Nat Protoc. 2018:13:377-91.

28. Mrdjen D, Pavlovic A, Hartmann FJ, Schreiner B, Utz SG, Leung BP, Lelios I, Heppner FL, Kipnis J, Merkler D, et al. High-dimensional single-cell mapping of central nervous system immune cells reveals distinct myeloid subsets in health, aging, and disease. Immunity. 2018:48:380-95.

29. Ornatsky OI, Lou X, Nitz M, Schafer S, Sheldrick WS, Baranov VI, Bandura DR, Tanner SD. Study of cell antigens and intracellular DNA by identification of element-containing labels and metallointercalators using inductively coupled plasma mass spectrometry. Anal Chem. 2008;80:2539-47.

30. Wlodarczyk A, Cedile O, Jensen KN, Jasson A, Mony JT, Khorooshi R, Owens T. Pathologic and protective roles for microglial subsets and bone marrowand blood-derived myeloid cells in central nervous system inflammation. Front Immunol. 2015;6:463. 
31. Cardona AE, Pioro EP, Sasse ME, Kostenko V, Cardona SM, Dijkstra IM, Huang D, Kidd G, Dombrowski S, Dutta R, et al. Control of microglial neurotoxicity by the fractalkine receptor. Nat Neurosci. 2006;9:917-24.

32. Garcia JA, Pino PA, Mizutani M, Cardona SM, Charo IF, Ransohoff RM, Forsthuber TG, Cardona AE. Regulation of adaptive immunity by the fractalkine receptor during autoimmune inflammation. J Immunol. 2013;191: 1063-72.

33. Sheridan GK, Murphy KJ. Neuron-glia crosstalk in health and disease: fractalkine and CX3CR1 take centre stage. Open Biol. 2013;3:130181.

34. Kiray H, Lindsay SL, Hosseinzadeh S, Barnett SC. The multifaceted role of astrocytes in regulating myelination. Exp Neurol. 2016;283:541-9.

35. Kawanokuchi J, Shimizu K, Nitta A, Yamada K, Mizuno T, Takeuchi H, Suzumura A. Production and functions of IL-17 in microglia. J Neuroimmunol. 2008;194:54-61.

36. Tzartos JS, Friese MA, Craner MJ, Palace J, Newcombe J, Esiri MM, Fugger L. Interleukin-17 production in central nervous system-infiltrating $T$ cells and glial cells is associated with active disease in multiple sclerosis. Am J Pathol. 2008;172:146-55

37. Hu MH, Zheng $Q F$, Jia $X Z$, Li Y, Dong $Y C$, Wang $C Y$, Lin $Q Y$, Zhang FY, Zhao RB, Xu HW, et al. Neuroprotection effect of interleukin (IL)-17 secreted by reactive astrocytes is emerged from a high-level IL-17-containing environment during acute neuroinflammation. Clin Exp Immunol. 2014;175: 268-84.

38. Erta M, Giralt M, Jimenez S, Molinero A, Comes G, Hidalgo J. Astrocytic IL-6 Influences the Clinical Symptoms of EAE in Mice. Brain Sci. 2016;6.

39. Schonrock LM, Gawlowski G, Bruck W. Interleukin-6 expression in human multiple sclerosis lesions. Neurosci Lett. 2000;294:45-8.

40. Brambilla R. The contribution of astrocytes to the neuroinflammatory response in multiple sclerosis and experimental autoimmune encephalomyelitis. Acta Neuropathol. 2019;137:757-83.

41. Lin W, Kemper A, Dupree JL, Harding HP, Ron D, Popko B. Interferongamma inhibits central nervous system remyelination through a process modulated by endoplasmic reticulum stress. Brain. 2006;129:1306-18.

42. Mendel I, Katz A, Kozak N, Ben-Nun A, Revel M. Interleukin-6 functions in autoimmune encephalomyelitis: a study in gene-targeted mice. Eur J Immunol. 1998;28:1727-37.

43. Cekanaviciute E, Fathali N, Doyle KP, Williams AM, Han J, Buckwalter MS. Astrocytic transforming growth factor-beta signaling reduces subacute neuroinflammation after stroke in mice. Glia. 2014;62:1227-40.

44. Yi W, Schluter D, Wang X. Astrocytes in multiple sclerosis and experimental autoimmune encephalomyelitis: Star-shaped cells illuminating the darkness of CNS autoimmunity. Brain Behav Immun. 2019;80:10-24.

\section{Publisher's Note}

Springer Nature remains neutral with regard to jurisdictional claims in published maps and institutional affiliations.

Ready to submit your research? Choose BMC and benefit from:

- fast, convenient online submission

- thorough peer review by experienced researchers in your field

- rapid publication on acceptance

- support for research data, including large and complex data types

- gold Open Access which fosters wider collaboration and increased citations

- maximum visibility for your research: over $100 \mathrm{M}$ website views per year

At BMC, research is always in progress.

Learn more biomedcentral.com/submissions 